Pure and Applied Mathematics Quarterly

Volume 3, Number 2

(Special Issue: In honor of

Leon Simon, Part 1 of 2)

$539-567,2007$

\title{
A Variational Formula for Stochastic Controls and Some Applications
}

\author{
Libin Mou and Jiongmin Yong* \\ For Honoring Professor Leon Simon on the Occasion of his 60-th Birthday
}

\begin{abstract}
For a controlled stochastic differential equation with a Bolza type performance functional, a variational formula for the functional in a given control process direction is derived, by means of backward stochastic differential equations. As applications, some Pontryagin type maximum principles are established for optimal controls of control problems, for saddle points of open-loop two-person zero-sum differential games, and for Nash equilibria of $N$-person nonzero-sum differential games. The results presented in this paper generalizes/simplifies the relevant ones found in [12] [17]. In addition, a sufficient existence condition of Nash equilibria is proved for nonzero-sum games.
\end{abstract}

Key words. Stochastic controls, variational formula, maximum principle, differential games, minimax principle, sufficient condition, saddle point, Nash equilibrium

AMS (MOS) subject classification. 49K, 93E20, 91A15, 91A23

\section{INTRODUCTION.}

Let $(\Omega, \mathcal{F}, \mathbb{F}, \mathbb{P})$ be a complete filtered probability space, on which a $d$-dimensional standard Brownian motion $W(\cdot)$ is defined with $\mathbb{F}=\left\{\mathcal{F}_{t}\right\}_{t \geq 0}$ being its natural filtration, augmented by all $\mathbb{P}$-null sets in $\mathcal{F}$. We consider a controlled stochastic differential equation (SDE, for short):

$$
\left\{\begin{array}{l}
d x(t)=b(t, x(t), u(t)) d t+\sigma(t, x(t), u(t)) d W(t), \quad t \in[0, T], \\
x(0)=x_{0},
\end{array}\right.
$$

Received December 31, 2005.

${ }^{*}$ This author was partially supported by NSF Grant DMS-0604309. 
and a performance functional vector

$$
J(u(\cdot))=\mathbb{E}\left\{\int_{0}^{T} f(t, x(t), u(t)) d t+h(x(T))\right\},
$$

where $b, \sigma, f$, and $h$ are given maps taking values in Euclidean spaces $\mathbb{R}^{n}, \mathbb{R}^{n \times d}$, $\mathbb{R}^{n}$, and $\mathbb{R}^{n}$, respectively. More assumptions will be made in the next section. In the above, $x(\cdot)$ is the (controlled) state process valued in $\mathbb{R}^{n}$, and $u(\cdot)$ is the control process valued in some set $U \subseteq \mathbb{R}^{m}$ (bounded or unbounded). Let us indicate some special cases of the above setting. When $N=1$, one can formulate an optimal control problem with state equation (1.1) and cost/payoff functional (1.2). When $N \geq 2$, $u(\cdot)=\left(u_{1}(\cdot), \cdots, u_{N}(\cdot)\right)$ with $u_{\ell}(\cdot)$ being the control of the $\ell$-th player, valued in $U_{\ell} \subseteq \mathbb{R}^{m_{\ell}}$ (with $m_{1}+\cdots+m_{N}=m$ ), which is to be chosen to optimize $J_{\ell}(u(\cdot))$, then one has an $N$-person (possibly) nonzero-sum differential game. If $N=2$ and

$$
J_{1}(u(\cdot))+J_{2}(u(\cdot))=0, \quad \forall u(\cdot),
$$

then one has a two-person zero-sum differential game. In addition, (1.1)-(1.2) covers the so-called multi-objective problems (which will be carefully discussed in a forthcoming paper). Indeed, (1.1)-(1.2) gives a very general model for a wide class of (stochastic) optimization problems. For optimal control problems, zero-sum or nonzero-sum differential games, one is mainly interested in optimal controls, saddle points, and Nash (or other type) equilibria. We may generally refer to them as extreme controls.

The purpose of this paper is to present necessary conditions for extreme controls in a unified way. The main idea is to derive a variational formula for the performance functional vector in certain given "control process direction", by means of backward stochastic differential equations (BSDEs, for short) and duality principle. Since $U$ may be non-convex, spike variations have to be used as the perturbation of the control process. A little different from [12] and [17], neither the variational systems nor the corresponding Taylor type expansions of the state process and the performance functional (with respect to a control variation) will be used. Instead, we first express the change of the performance functional directly in terms of the Hamiltonian and associated first order and second order adjoint systems (which are BSDEs). Then we obtain a representation for the variation and "directional derivative" of the performance functional. This idea of using the adjoint systems alone is very natural because the statement of Pontryagin type maximum principle involves only the adjoint systems, not the variational systems. Similar idea is used in [15] for optimal control of variational inequalities.

This paper is organized as follows. The assumptions, notations and some basic estimates (Proposition 2.1, Corollary 2.1) are given in Section 2. In Section 3, we obtain two representations (first order and second order) for the difference of the performance functional in terms of the Hamiltonian and adjoint processes (Lemma 3.1). These representations may be of interest in their own right. In Section 4 we use the second order representation in Section 3 to derive a representation for the variation and "directional derivative" of the performance functional along with any spike variation (Theorem 4.1). 
In Section 5, we apply the "directional derivative" formula from Section 4 to generalize the classical maximum principle for stochastic optimal controls (Theorem 5.1) and derive extreme principles for Nash equilibria of nonzero-sum games (Theorem 5.2) and minimax principle for saddle points of a zero-sum game (Theorem 5.3). In Section 6, we use the first order difference representation in Section 3 to obtain a sufficient condition for Nash equilibria of a nonzero-sum game (Theorem 6.2).

Theorem 6.2 shows that open-loop Nash equilibria exist under reasonable conditions. While equilibria of other types (closed-loop, strategies, etc.) are commonly considered for differential games, all of them (if exist) induce open-loop controls. A natural question is whether they induce open-loop Nash equilibria. This question is one of our motivations for studying open-loop equilibria of differential games; see [11] and [16] for some recent results on linear-quadratic stochastic games.

\section{Assumptions and Basic Estimates}

In this section we will make some preliminaries. First of all, besides the Euclidean space $\mathbb{R}^{n}$ and the matrix space $\mathbb{R}^{n \times d}$ (of all $(n \times d)$ matrices), we let $\mathcal{S}^{n}$ be the set of all $(n \times n)$ symmetric matrices. For any $x, y \in \mathbb{R}^{n}$, we use $x^{T} y$ and $x \cdot y$ to denote the inner product of these two vectors. For a function $\varphi: \mathbb{R}^{n} \rightarrow \mathbb{R}$, denote by $\nabla_{x} \varphi=\varphi_{x}$ its gradient (as a column vector) and $\nabla_{x}^{2} \varphi=\varphi_{x x}$ its Hessian (a symmetric matrix). If $\varphi: \mathbb{R}^{n} \rightarrow \mathbb{R}^{k}$ (with $k \geq 2$ ), then $\varphi_{x}=\left(\frac{\partial \varphi_{i}}{\partial x_{j}}\right)_{1 \leq i \leq k, 1 \leq j \leq n}$ is the corresponding $(k \times n)$ Jacobian matrix. In what follows, $C$ represents a generic constant, which can be different from line to line.

Next we introduce some spaces of random variables and stochastic processes. For any $\alpha, \beta \in[1, \infty)$, we let

$$
\begin{aligned}
& L_{\mathcal{F}_{T}}^{\beta}\left(\Omega ; \mathbb{R}^{n}\right) \triangleq\left\{\xi: \Omega \rightarrow \mathbb{R}^{n} \mid \xi \text { is } \mathcal{F}_{T^{-}} \text {-measurable, } \mathbb{E}|\xi|^{\beta}<\infty\right\}, \\
& L_{\mathcal{F}}^{\beta}\left(0, T ; \mathbb{R}^{k}\right) \triangleq\left\{\varphi:[0, T] \times \Omega \rightarrow \mathbb{R}^{k} \mid \varphi(\cdot) \text { is } \mathbb{F} \text {-adapted, } \mathbb{E} \int_{0}^{T}|\varphi(t)|^{\beta} d t<\infty\right\}, \\
& L_{\mathcal{F}}^{\beta}\left(\Omega ; L^{\alpha}\left(0, T ; \mathbb{R}^{k}\right)\right) \triangleq\left\{\varphi:[0, T] \times \Omega \rightarrow \mathbb{R}^{k} \mid \varphi(\cdot) \text { is } \mathbb{F} \text {-adapted, }\|\varphi\|_{\alpha, \beta}<\infty\right\}, \\
& L_{\mathcal{F}}^{\beta}\left(\Omega ; C\left([0, T] ; \mathbb{R}^{k}\right)\right) \triangleq\left\{\varphi:[0, T] \times \Omega \rightarrow \mathbb{R}^{k} \mid \varphi(\cdot) \text { is } \mathbb{F}\right. \text {-adapted, } \\
& \text { has continuous paths, } \left.\|\varphi\|_{\infty, \beta}<\infty\right\},
\end{aligned}
$$

where $\|\varphi\|_{\alpha, \beta}$ and $\|\varphi\|_{\infty, \beta}$ are norms defined as

$$
\|\varphi\|_{\alpha, \beta} \triangleq\left[\mathbb{E}\left(\int_{0}^{T}|\varphi(t)|^{\alpha} d t\right)^{\frac{\beta}{\alpha}}\right]^{\frac{1}{\beta}} ; \quad\|\varphi\|_{\infty, \beta} \triangleq\left[\mathbb{E}\left(\sup _{t \in[0, T]}|\varphi(t)|^{\beta}\right)\right]^{\frac{1}{\beta}} .
$$


For a subset $U \in \mathbb{R}^{m}$, we define

$$
\begin{aligned}
& L_{\mathcal{F}}^{\beta}\left(\Omega ; L^{\alpha}(0, T ; U)\right) \triangleq\left\{u(\cdot) \in L_{\mathcal{F}}^{\beta}\left(\Omega ; L^{\alpha}\left(0, T ; \mathbb{R}^{m}\right)\right) \mid u(t) \in U, \text { a.e., a.s. }\right\}, \\
& L_{\mathcal{F}}^{\beta}(0, T ; U) \triangleq\left\{u(\cdot) \in L_{\mathcal{F}}^{\beta}\left(0, T ; \mathbb{R}^{m}\right) \mid u(t) \in U \text {, a.e., a.s. }\right\} .
\end{aligned}
$$

Now we introduce the following standing assumptions.

(S1) The control space $U \subseteq \mathbb{R}^{m}$ is nonempty.

(S2) Maps b: $[0, T] \times \mathbb{R}^{n} \times U \times \Omega \rightarrow \mathbb{R}^{n}, \sigma:[0, T] \times \mathbb{R}^{n} \times U \times \Omega \rightarrow \mathbb{R}^{n \times d}$, $f:[0, T] \times \mathbb{R}^{n} \times U \times \Omega \rightarrow \mathbb{R}^{n}$, and $h: \mathbb{R}^{n} \times \Omega \rightarrow \mathbb{R}^{n}$ satisfy the following:

(i) $b, \sigma, f$ are $\mathcal{B}\left([0, T] \times \mathbb{R}^{n} \times U\right) \otimes \mathcal{F}_{T}$-measurable, and $h$ is $\mathcal{B}\left(\mathbb{R}^{n}\right) \otimes \mathcal{F}_{T}$-measurable, where $\mathcal{B}(G)$ is the Borel $\sigma$-field of the metric space $G$.

(ii) For each $(x, u) \in \mathbb{R}^{n} \times U, t \mapsto(b(t, x, u), \sigma(t, x, u), f(t, x, u))$ is $\mathbb{F}$-adapted.

(iii) For almost all $(t, u, \omega) \in[0, T] \times \mathbb{R}^{n} \times \Omega$, the map

$$
x \mapsto(b(t, x, u, \omega), \sigma(t, x, u, \omega), f(t, x, u, \omega), h(x, \omega))
$$

is twice continuously differentiable with all the partial derivatives of $b, \sigma, f$, and $h$ with respect to $x$ up to order 2 being continuous in $(x, u)$ with appropriate growths. More precisely, there exist a constant $L>0$, a process $\rho(\cdot) \in L_{\mathcal{F}}^{\beta}\left(\Omega ; L^{2}(0, T ; \mathbb{R})\right)($ with $\beta \geq 1)$ and a modulus of continuity $\widetilde{\omega}:[0, \infty) \rightarrow[0,1]$ such that for $\varphi=b^{j}, \sigma^{i j}(1 \leq i \leq d$, $1 \leq j \leq n)$,

$$
\left\{\begin{array}{l}
|\varphi(t, x, u)| \leq L[\rho(t)+|x|+|u|] \\
\left|\varphi_{x}(t, x, u)\right|,\left|\varphi_{x x}(t, x, u)\right| \leq L \\
\left|\varphi_{x x}(t, x, u)-\varphi_{x x}(t, \widetilde{x}, u)\right| \leq L \widetilde{\omega}(|x-\widetilde{x}|)
\end{array}\right.
$$

and for $1 \leq \ell \leq N$,

$$
\left\{\begin{array}{l}
\left|\nabla_{x}^{k} f^{\ell}(t, x, u)\right| \leq L[\rho(t)+|x|+|u|]^{2-k} \text { for } k=0,1,2 \\
\left|f_{x x}^{\ell}(t, x, u)-f_{x x}^{\ell}(t, \widetilde{x}, u)\right| \leq L \widetilde{\omega}(|x-\widetilde{x}|) \\
\left|\nabla_{x}^{k} h^{\ell}(x)\right| \leq L(\rho(T)+|x|)^{2-k} \text { for } k=0,1,2 \\
\left|h_{x x}^{\ell}(x)-h_{x x}^{\ell}(\widetilde{x})\right| \leq L \widetilde{\omega}(|x-\widetilde{x}|)
\end{array}\right.
$$

The set of admissible controls is defined as

$$
\mathcal{U}^{\beta}[0, T] \triangleq L_{\mathcal{F}}^{\beta}\left(\Omega ; L^{2}(0, T ; U)\right) .
$$

The following result gives the well-posedness of the state equation as well as some useful estimates. 
Proposition 2.1. Let (S1)-(S2) hold. Then for any $u(\cdot) \in \mathcal{U}^{\beta}[0, T]$ with $\beta \geq 1$, state equation (1.1) admits a unique strong solution $x(\cdot) \in L_{\mathcal{F}}^{\beta}\left(\Omega ; C\left([0, T] ; \mathbb{R}^{n}\right)\right)$, and the following estimate holds:

$$
\begin{aligned}
& \mathbb{E}\left[\sup _{s \in[0, t]}|x(s)|^{\beta}\right] \\
& \leq C \mathbb{E}\left\{\left|x_{0}\right|^{\beta}+\left(\int_{0}^{t}|b(s, 0, u(s))| d s\right)^{\beta}+\left(\int_{0}^{t}|\sigma(s, 0, u(s))|^{2} d s\right)^{\frac{\beta}{2}}\right\} \\
& \leq C \mathbb{E}\left\{\left|x_{0}\right|^{\beta}+\left(\int_{0}^{t}|\rho(s)|^{2} d s\right)^{\frac{\beta}{2}}+\left(\int_{0}^{t}|u(s)|^{2} d s\right)^{\frac{\beta}{2}}\right\} .
\end{aligned}
$$

Further, if $\bar{x}(\cdot)$ is the unique solution corresponding to $\left(\bar{x}_{0}, \bar{u}(\cdot)\right) \in \mathbb{R}^{n} \times \mathcal{U}^{\beta}[0, T]$, then

$$
\begin{aligned}
& \mathbb{E}\left[\sup _{s \in[0, t]}|x(s)-\bar{x}(s)|^{\gamma}\right] \\
& \leq C\left[\left|x_{0}-\bar{x}_{0}\right|^{\gamma}+E\left(\int_{0}^{t}|b(s, \bar{x}(s), u(s))-b(s, \bar{x}(s), \bar{u}(s))| d s\right)^{\gamma}\right. \\
& \left.+E\left(\int_{0}^{t}|\sigma(s, \bar{x}(s), u(s))-\sigma(s, \bar{x}(s), \bar{u}(s))|^{2} d s\right)^{\frac{\gamma}{2}}\right],
\end{aligned}
$$

for all $\gamma \geq 1$.

Proof. The existence and uniqueness of the strong solution to (1.1) follows from the contraction mapping theorem in the usual manner. We now prove (2.3). Suppose $\left(x_{0}, u(\cdot)\right) \in \mathbb{R}^{n} \times \mathcal{U}^{\beta}[0, T]$ is given and $x(\cdot)$ is the strong solution of (1.1). By assumption (S2), $|\varphi(t, x, u)| \leq|\varphi(t, 0, u)|+L|x|$ for $\varphi=b$ and $\sigma$. Then by Burkholder-DavisGundy's inequality,

$$
\begin{aligned}
& \mathbb{E}\left[\sup _{s \in[0, t]}|x(s)|^{\beta}\right] \\
& \leq C \mathbb{E}\left\{\left|x_{0}\right|^{\beta}+\left(\int_{0}^{t}|b(s, x(s), u(s))| d s\right)^{\beta}+\left(\int_{0}^{t}|\sigma(s, x(s), u(s))|^{2} d s\right)^{\frac{\beta}{2}}\right\} \\
& \leq C \mathbb{E}\left\{\left|x_{0}\right|^{\beta}+\left(\int_{0}^{t}|b(s, 0, u(s))| d s\right)^{\beta}+\left(\int_{0}^{t}|\sigma(s, 0, u(s))|^{2} d s\right)^{\frac{\beta}{2}}+\left(\int_{0}^{t}|x(s)|^{2} d s\right)^{\frac{\beta}{2}}\right\} \\
& \leq C \mathbb{E}\left\{\left|x_{0}\right|^{\beta}+\left(\int_{0}^{t}|b(s, 0, u(s))| d s\right)^{\beta}+\left(\int_{0}^{t}|\sigma(s, 0, u(s))|^{2} d s\right)^{\frac{\beta}{2}}+t^{\frac{\beta}{2}} \sup _{s \in[0, t]}|x(s)|^{\beta}\right\} .
\end{aligned}
$$

Note that the constant $C>0$ in (2.5) depends on $\beta$ and the constant $L$ from (S2) only, not on the interval $[0, t]$. By taking $\delta=(2 C)^{-\frac{2}{\beta}}$, we have

$$
\begin{aligned}
& \mathbb{E}\left[\sup _{s \in[0, \delta]}|x(s)|^{\beta}\right] \\
& \leq 2 C \mathbb{E}\left\{\left|x_{0}\right|^{\beta}+\left(\int_{0}^{\delta}|b(s, 0, u(s))| d s\right)^{\beta}+\left(\int_{0}^{\delta}|\sigma(s, 0, u(s))|^{2} d s\right)^{\frac{\beta}{2}}\right\} .
\end{aligned}
$$


Next, on $[\delta, 2 \delta]$, similar to the above, we have

$$
\begin{aligned}
& \mathbb{E}\left[\sup _{s \in[\delta, 2 \delta]}|x(s)|^{\beta}\right] \\
& \leq C \mathbb{E}\left\{|x(\delta)|^{\beta}+\left(\int_{\delta}^{2 \delta}|b(s, 0, u(s))| d s\right)^{\beta}+\left(\int_{\delta}^{2 \delta}|\sigma(s, 0, u(s))|^{2} d s\right)^{\frac{\beta}{2}}\right. \\
& \left.+\delta^{\frac{\beta}{2}} \sup _{s \in[\delta, 2 \delta]}|x(s)|^{\beta}\right\},
\end{aligned}
$$

where the constant $C$ in (2.7) is the same as that in (2.5). Hence, we obtain

$$
\begin{aligned}
& \mathbb{E}\left[\sup _{s \in[\delta, 2 \delta]}|x(s)|^{\beta}\right] \\
& \leq 2 C \mathbb{E}\left\{|x(\delta)|^{\beta}+\left(\int_{\delta}^{2 \delta}|b(s, 0, u(s))| d s\right)^{\beta}+\left(\int_{\delta}^{2 \delta}|\sigma(s, 0, u(s))|^{2} d s\right)^{\frac{\beta}{2}}\right\} .
\end{aligned}
$$

Since the step-length $\delta>0$ will not shrink, by induction, we obtain (2.3).

Note that under (iii) of (S2), we have

$$
|b(t, 0, u)|+|\sigma(t, 0, u)| \leq 2 L(\rho(t)+|u|) .
$$

So the second inequality (2.3) follows.

In the same fashion, we can prove (2.4).

Note that for any $u(\cdot) \in \mathcal{U}^{\beta}[0, T]$ (with $\beta \geq 1$ ), the right hand side of (2.3) is finite. By assumption (S2), we have

$$
\left\{\begin{array}{l}
|h(x(T))| \leq L(\rho(T)+|x(T)|)^{2} \\
|f(t, x(t), u(t))| \leq L(\rho(t)+|x(t)|+|u(t)|)^{2} .
\end{array}\right.
$$

Hence, combining Proposition 2.1 with the assumption on $f$ and $h$, we see that for any $\beta \geq 2$, the performance functional vector $J(u(\cdot))$ is well-defined on $\mathcal{U}^{\beta}[0, T]$.

Note that

$$
\begin{cases}L_{\mathcal{F}}^{\beta}(0, T ; U) \subseteq \mathcal{U}^{\beta}[0, T], & \beta>2, \\ L_{\mathcal{F}}^{\beta}(0, T ; U) \supseteq \mathcal{U}^{\beta}[0, T], & \beta<2, \\ L_{\mathcal{F}}^{\beta}(0, T ; U)=\mathcal{U}^{\beta}[0, T], & \beta=2 .\end{cases}
$$

So the performance functional vector $J(u(\cdot))$ is also well-defined on $L_{\mathcal{F}}^{\beta}(0, T ; U)$ for $\beta \geq 2$. While we will focus on the admissible set $\mathcal{U}^{\beta}[0, T]$ in this paper, all of the results obtained in this paper for $\mathcal{U}^{\beta}[0, T]$ also hold for $L_{\mathcal{F}}^{\beta}(0, T ; U)$ with $\beta \geq 2$.

The estimate (2.4) show that although we might only have $\bar{x}(\cdot) \in L_{\mathcal{F}}^{\beta}\left(\Omega ; C\left([0, T] ; \mathbb{R}^{n}\right)\right)$ for $\bar{u}(\cdot) \in \mathcal{U}^{\beta}[0, T]$, the change of the state (the left hand side of (2.4)) is controlled by the perturbation of $\bar{u}(\cdot)$ to $u(\cdot)$. Here $\gamma$ might be larger than $\beta$.

From the estimates in Proposition 2.1, we obtain 
Corollary 2.1. Let $(\bar{x}(\cdot), \bar{u}(\cdot))$ and $(x(\cdot), u(\cdot))$ be two admissible pairs, and $\Delta x(\cdot)=$ $x(\cdot)-\bar{x}(\cdot)$. Then there exist a constant $C>0$ independent on $\bar{u}(\cdot)$ and $u(\cdot)$ such that

$$
\begin{aligned}
& \|\bar{x}(\cdot)\|_{\infty, \beta} \leq C\left[\|\bar{x}(0)\|_{\infty, \beta}+\|\rho(\cdot)\|_{2, \beta}+\|\bar{u}(\cdot)\|_{2, \beta}\right], \\
& \|\Delta x(\cdot)\|_{\infty, \beta} \leq C\left[\|\Delta x(0)\|_{\infty, \beta}+\left\|(2|\bar{x}|+2 \rho+|\bar{u}|+|u|) I_{\{u \neq \bar{u}\}}\right\|_{2, \beta}\right],
\end{aligned}
$$

where $\|\bar{x}(0)\|_{\infty, \beta}=\left[\mathbb{E}|\bar{x}(0)|^{\beta}\right]^{\frac{1}{\beta}}$, and $I_{\{u \neq \bar{u}\}}(t, \omega)=1$ if $u(t, \omega)=\bar{u}(t, \omega)$ and 0 otherwise.

Proof. The first inequality follows from (2.3) written in norms. To prove the second inequality, we use (2.4). By assumption (S2), we have that for $\varphi=b$ and $\sigma$,

$$
|\varphi(t, \bar{x}(t), u(t))-\varphi(t, \bar{x}(t), \bar{u}(t))| \leq L[2 \rho(t)+2|\bar{x}(t)|+|\bar{u}(t)|+|u(t)|] I_{\{u \neq \bar{u}\}} .
$$

By (2.4) in Proposition 2.1 with $\gamma=\beta$,

$$
\begin{aligned}
& \|\Delta x(\cdot)\|_{\infty, \beta}=\mathbb{E}\left[\sup _{t \in[0, T]}|\Delta x(t)|^{\beta}\right]^{\frac{1}{\beta}} \\
& \leq C \mathbb{E}\left[|\Delta x(0)|^{\beta}+\left(\int_{0}^{T}\left|\Delta_{u} b(t, u(t))\right| d t\right)^{\beta}+\left(\int_{0}^{T}\left|\Delta_{u} \sigma(t, u(t))\right|^{2}(t) d t\right)^{\frac{\beta}{2}}\right]^{\frac{1}{\beta}} \\
& \leq C\left[\|\Delta x(0)\|_{\infty, \beta}+\left\|(2|\bar{x}|+2 \rho+|\bar{u}|+|u|) I_{\{u \neq \bar{u}\}}\right\|_{2, \beta}\right] .
\end{aligned}
$$

\section{Representations for Difference of the Performance Functional} VECTOR

For given maps $b, \sigma, f$, and $h$ satisfying (S1)-(S2), we define the associated Hamiltonian as follows:

$$
\begin{array}{r}
H^{\ell}\left(t, x, u, p^{\ell}, q^{\ell}\right)=f^{\ell}(t, x, u)+p^{\ell} \cdot b(t, x, u)+\sum_{i=1}^{d} q_{i}^{\ell} \cdot \sigma^{i}(t, x, u), \\
\left(t, x, u, p^{\ell}, q^{\ell}\right) \in[0, T] \times \mathbb{R}^{n} \times U \times \mathbb{R}^{n} \times \mathbb{R}^{n \times d}, \quad 1 \leq \ell \leq N .
\end{array}
$$

For any given admissible pair $(\bar{x}(\cdot), \bar{u}(\cdot))$, we introduce the following system of linear BSDEs:

$$
\left\{\begin{array}{l}
d p^{\ell}(t)=-H_{x}^{\ell}\left(t, \bar{x}(t), \bar{u}(t), p^{\ell}(t), q^{\ell}(t)\right) d t+q^{\ell}(t) d W(t), \\
p^{\ell}(T)=h_{x}^{\ell}(\bar{x}(T)), \quad 1 \leq \ell \leq N,
\end{array}\right.
$$


and

(3.3)

$$
\left\{\begin{aligned}
d P^{\ell}(t)= & -\left\{H_{x x}^{\ell}(t)+P^{\ell}(t) b_{x}(t)+b_{x}(t)^{T} P^{\ell}(t)\right. \\
& \left.+\sum_{i=1}^{d}\left[\sigma_{x}^{i}(t)^{T} P^{\ell}(t) \sigma_{x}^{i}(t)+Q_{i}^{\ell}(t) \sigma_{x}^{i}(t)+\sigma_{x}^{i}(t)^{T} Q_{i}^{\ell}(t)\right]\right\} d t+\sum_{i=1}^{d} Q_{i}^{\ell}(t) d W_{i}(t), \\
P^{\ell}(T)= & h_{x x}^{\ell}(\bar{x}(T)), \quad 1 \leq \ell \leq N,
\end{aligned}\right.
$$

where

$$
\left\{\begin{array}{l}
b_{x}(t)=b_{x}(t, \bar{x}(t), \bar{u}(t)), \quad \sigma_{x}^{i}(t)=\sigma_{x}^{i}(t, \bar{x}(t), \bar{u}(t)), \quad 1 \leq i \leq d, \\
H_{x x}^{\ell}(t)=H_{x x}^{\ell}\left(t, \bar{x}(t), \bar{u}(t), p^{\ell}(t), q^{\ell}(t)\right)
\end{array}\right.
$$

with $\left\{\left(p^{\ell}(\cdot), q^{\ell}(\cdot)\right), 1 \leq \ell \leq N\right\}$ being the (unique) adapted solution of (3.2). We call (3.2) and (3.3) the first and the second adjoint equations of (1.1)-(1.2), respectively. We also refer to the adapted solutions $\left\{\left(p^{\ell}(\cdot), q^{\ell}(\cdot)\right), 1 \leq \ell \leq N\right\}$ of $(3.2)$ and $\left\{\left(P^{\ell}(\cdot), Q^{\ell}(\cdot)\right), 1 \leq \ell \leq N\right\}$ of (3.3) as the first order and the second order adjoint processes associated with $(\bar{x}(\cdot), \bar{u}(\cdot))$, respectively. We have the following result concerning the unique solvability of (3.2) and (3.3).

Proposition 3.1. Let (S1)-(S2) hold and $(\bar{x}(\cdot), \bar{u}(\cdot))$ be an admissible pair with $\bar{u}(\cdot) \in$ $\mathcal{U}^{\beta}[0, T](\beta>1)$. Then (3.2) and (3.3) admit unique adapted solutions, respectively. Moreover, the following estimates hold:

$$
\mathbb{E}\left[\sup _{t \in[0, T]}\left|P^{\ell}(t)\right|^{\beta}\right]+\sum_{i=1}^{d} \mathbb{E}\left[\int_{0}^{T}\left|Q_{i}^{\ell}(t)\right|^{2} d t\right]^{\frac{\beta}{2}} \leq C\left\{1+\left(\int_{0}^{T}|\bar{u}(s)|^{2} d s\right)^{\frac{\beta}{2}}\right\}
$$

Proof. By the definition of $H^{\ell}$, we have

$$
\begin{aligned}
& H_{x}^{\ell}\left(t, \bar{x}(t), \bar{u}(t), p^{\ell}(t), q^{\ell}(t)\right) \\
& =b_{x}(t, \bar{x}(t), \bar{u}(t)) p^{\ell}(t)+\sum_{i=1}^{d} \sigma_{x}^{i}(t, \bar{x}(t), \bar{u}(t)) q_{i}^{\ell}(t)+f_{x}^{\ell}(t, \bar{x}(t), \bar{u}(t)) .
\end{aligned}
$$


Hence, by (S2) and [6], we can find a unique adapted solution $\left\{\left(p^{\ell}(\cdot), q^{\ell}(\cdot)\right), 1 \leq \ell \leq N\right\}$ to (3.2). Moreover,

$$
\begin{aligned}
& \mathbb{E}\left[\sup _{t \in[0, T]}\left|p^{\ell}(t)\right|^{\beta}\right]+\mathbb{E}\left[\int_{0}^{T}\left|q^{\ell}(t)\right|^{2} d t\right]^{\frac{\beta}{2}} \\
& \leq C \mathbb{E}\left\{\left|h_{x}^{\ell}(\bar{x}(T))\right|^{\beta}+\left(\int_{0}^{T}\left|f_{x}^{\ell}(t, \bar{x}(t), \bar{u}(t))\right| d t\right)^{\beta}\right\} \\
& \leq C\left\{1+\mathbb{E}|\bar{x}(T)|^{\beta}+\mathbb{E}\left[\int_{0}^{T}(\rho(t)+|\bar{x}(t)|+|\bar{u}(t)|) d t\right]^{\beta}\right\} \\
& \leq C\left\{1+\mathbb{E}\left(\int_{0}^{T}|\bar{u}(s)|^{2} d s\right)^{\frac{\beta}{2}}\right\} .
\end{aligned}
$$

This proves estimate (3.5).

Next, by (S2), we have

$$
\left\{\begin{array}{l}
\left|h_{x x}^{\ell}(\bar{x}(T))\right| \leq L, \\
\left|H_{x x}^{\ell}(t)\right| \leq L\left(1+\left|p^{\ell}(t)\right|+\left|q^{\ell}(t)\right|\right) .
\end{array}\right.
$$

Hence, by [6], we have the existence and uniqueness of adapted solution to (3.3). Moreover,

$$
\begin{aligned}
& \mathbb{E}\left[\sup _{t \in[0, T]}\left|P^{\ell}(t)\right|^{\beta}+\sum_{i=1}^{d} \mathbb{E}\left(\int_{0}^{T}\left|Q_{i}^{\ell}(t)\right|^{2} d t\right)^{\frac{\beta}{2}}\right] \\
& \leq C \mathbb{E}\left\{\left|h_{x x}^{\ell}(\bar{x}(T))\right|^{\beta}+\left(\int_{0}^{T}\left|H_{x x}^{\ell}(t)\right| d t\right)^{\beta}\right\} \\
& \leq C \mathbb{E}\left\{1+\left[\int_{0}^{T}\left(\left|p^{\ell}(t)\right|+\left|q^{\ell}(t)\right|\right) d t\right]^{\beta}\right\} \\
& \leq C\left[1+\mathbb{E}\left(\int_{0}^{T}|\bar{u}(t)|^{2} d t\right)^{\frac{\beta}{2}}\right] .
\end{aligned}
$$

This proves (3.6).

We point out that for $N \geq 2$, when the admissible pair $(\bar{x}(\cdot), \bar{u}(\cdot))$ is given, BSDEs (3.2) consists of $N$ decoupled BSDEs. However, when $(\bar{u}(\cdot)) \equiv\left(\bar{u}_{1}(\cdot), \cdots, \bar{u}_{N}(\cdot)\right)$ is, say, a Nash equilibrium, then the corresponding optimality system will be a system of forward-backward stochastic differential equations (FBSDEs, for short), in which all the components will be coupled through the necessary conditions satisfied by the components $\bar{u}_{\ell}(\cdot)$ of $\bar{u}(\cdot)$. We will see this clearer later. The same remark applies to BSDE system (3.3).

Now let $\left(x_{\varepsilon}(\cdot), u_{\varepsilon}(\cdot)\right)$ be another admissible pair. Our first main result gives two representations for the difference $J\left(u_{\varepsilon}(\cdot)\right)-J(\bar{u}(\cdot))$ in terms of the Hamiltonian and 
adjoint processes associated with $(\bar{x}(\cdot), \bar{u}(\cdot))$, as well as other relevant expressions. To state the result, let us introduce the following notations:

$$
\left\{\begin{array}{l}
\Delta x(t)=x_{\varepsilon}(t)-\bar{x}(t), \quad t \in[0, T], \\
\Delta_{x}^{0} h^{\ell}(T)=h^{\ell}\left(x_{\varepsilon}(T)\right)-h^{\ell}(\bar{x}(T)), \\
\Delta_{x}^{1} h^{\ell}(T)=\Delta_{x} h^{\ell}(T)-h_{x}^{\ell}(\bar{x}(T))^{T} \Delta x(T), \\
\Delta_{x}^{2} h^{\ell}(T)=\Delta_{x}^{1} h^{\ell}(T)-\frac{1}{2} \Delta x(T)^{T} h_{x x}^{\ell}(\bar{x}(T)) \Delta x(T),
\end{array}\right.
$$

and

$$
\left\{\begin{array}{l}
\Delta_{x u}^{0} H^{\ell}(t)=H^{\ell}\left(t, x_{\varepsilon}(t), u_{\varepsilon}(t), p(t), q(t)\right)-H^{\ell}(t, \bar{x}(t), \bar{u}(t), p(t), q(t)) \\
\Delta_{x u}^{1} H^{\ell}(t)=\Delta_{x u} H^{\ell}(t)-H_{x}^{\ell}(t, \bar{x}(t), \bar{u}(t), p(t), q(t)) \cdot \Delta x(t) \\
\Delta_{x u}^{2} H^{\ell}(t)=\Delta_{x u}^{1} H^{\ell}(t)-\frac{1}{2} \Delta x(t)^{T} H_{x x}^{\ell}(t, \bar{x}(t), \bar{u}(t), p(t), q(t)) \Delta x(t) .
\end{array}\right.
$$

The definitions of $\Delta_{x u}^{k} f^{\ell}(t), \Delta_{x u}^{k} b(t)$, and $\Delta_{x u}^{k} \sigma^{i}(t)($ with $k=0,1,2)$ are similar to the above.

Lemma 3.1. Let (S1)-(S2) hold. Let $(\bar{x}(\cdot), \bar{u}(\cdot))$ be any admissible pair, whose associated Hamiltonian is $H$, and the associated first and second order adjoint processes are $\left\{\left(p^{\ell}(\cdot), q^{\ell}(\cdot)\right), 1 \leq \ell \leq N\right\}$ and $\left\{\left(P^{\ell}(\cdot), Q^{\ell}(\cdot)\right), 1 \leq \ell \leq N\right\}$. Let $\left(x_{\varepsilon}(\cdot), u_{\varepsilon}(\cdot)\right)$ be another admissible pair. Then for each $1 \leq \ell \leq N$,

$$
\begin{aligned}
& J^{\ell}\left(u_{\varepsilon}(\cdot)\right)-J^{\ell}(\bar{u}(\cdot)) \\
& =\mathbb{E}\left[\Delta_{x}^{1} h^{\ell}(T)+\int_{0}^{T} \Delta_{x u}^{1} H^{\ell}(t) d t\right] \\
& =\mathbb{E}\left\{\Delta_{x}^{2} h^{\ell}(T)+\int_{0}^{T}\left[\Delta_{x u}^{2} H^{\ell}(t)+\Delta x(t)^{T}\left(P^{\ell}(t) \Delta_{x u}^{1} b(t)+\sum_{i=1}^{d} Q_{i}^{\ell}(t) \Delta_{x u}^{1} \sigma^{i}(t)\right)\right.\right. \\
& \left.\left.\quad+\frac{1}{2} \sum_{i=1}^{d}\left(\Delta_{x u}^{0} \sigma^{i}(t)^{T} P^{\ell}(t) \Delta_{x u}^{0} \sigma^{i}(t)-\Delta x(t)^{T} \sigma_{x}^{i}(t)^{T} P^{\ell}(t) \sigma_{x}^{i}(t) \Delta x(t)\right)\right] d t\right\},
\end{aligned}
$$

where $\sigma_{x}^{i}(t)$ is the same as in (3.4).

We refer to the first equality in (3.13) as the first order representation, and to the second equality in (3.13) as the second order representation, respectively, for the difference $J\left(u_{\varepsilon}(\cdot)\right)-J(\bar{u}(\cdot))$. 
Proof. By (1.2), we have (note (3.11) and (3.12))

$$
\begin{aligned}
& J^{\ell}\left(u_{\varepsilon}(\cdot)\right)-J^{\ell}(\bar{u}(\cdot)) \\
& =\mathbb{E}\left\{h^{\ell}\left(x_{\varepsilon}(T)\right)-h^{\ell}(\bar{x}(T))+\int_{0}^{T}\left[f^{\ell}\left(t, x_{\varepsilon}(t), u_{\varepsilon}(t)\right)-f^{\ell}(t, \bar{x}(t), \bar{u}(t))\right] d t\right\} \\
& =\mathbb{E}\left[\Delta_{x}^{0} h^{\ell}(T)+\int_{0}^{T} \Delta_{x u}^{0} f^{\ell}(t) d t\right],
\end{aligned}
$$

Now we let $\left\{\left(p^{\ell}(\cdot), q^{\ell}(\cdot)\right), 1 \leq \ell \leq N\right\}$ be the adapted solution of (3.2). From (1.1), we know that

$$
d[\Delta x(t)]=\Delta_{x u}^{0} b(t) d t+\Delta_{x u}^{0} \sigma(t) d W(t), \quad \Delta x(0)=0 .
$$

Applying Itô's formula, we have (note (3.12))

$\mathbb{E}\left[h_{x}^{\ell}(\bar{x}(T)) \cdot \Delta x(T)\right]=\mathbb{E}\left[p^{\ell}(T) \cdot \Delta x(T)\right]$

$=\mathbb{E} \int_{0}^{T}\left[-H_{x}^{\ell}\left(t, \bar{x}(t), \bar{u}(t), p^{\ell}(t), q^{\ell}(t)\right) \cdot \Delta x(t)+p^{\ell}(t) \cdot \Delta_{x u}^{0} b(t)+\sum_{i=1}^{d} q_{i}^{\ell}(t) \cdot \Delta_{x u}^{0} \sigma^{i}(t)\right] d t$

$=\mathbb{E} \int_{0}^{T}\left[-H_{x}^{\ell}\left(t, \bar{x}(t), \bar{u}(t), p^{\ell}(t), q^{\ell}(t)\right) \cdot \Delta x(t)+\Delta_{x u}^{0} H^{\ell}(t)-\Delta_{x u}^{0} f^{\ell}(t)\right] d t$

$=\mathbb{E} \int_{0}^{T}\left[\Delta_{x u}^{1} H^{\ell}(t)-\Delta_{x u}^{0} f^{\ell}(t)\right] d t$.

Hence, by (3.14),

$$
\begin{aligned}
& J^{\ell}\left(u_{\varepsilon}(\cdot)\right)-J^{\ell}(\bar{u}(\cdot))=\mathbb{E}\left[\Delta_{x}^{0} h^{\ell}(T)+\int_{0}^{T} \Delta_{x u}^{0} f^{\ell}(t) d t\right] \\
& =\mathbb{E}\left[\Delta_{x}^{1} h^{\ell}(T)+h_{x}^{\ell}(\bar{x}(T)) \cdot \Delta x(T)+\int_{0}^{T} \Delta_{x u}^{0} f^{\ell}(t) d t\right] \\
& =\mathbb{E}\left[\Delta_{x}^{1} h^{\ell}(T)+\int_{0}^{T} \Delta_{x u}^{1} H^{\ell}(t) d t\right],
\end{aligned}
$$

proving the first equality in (3.13).

Next, we let $\left\{\left(P^{\ell}(\cdot), Q^{\ell}(\cdot)\right), 1 \leq \ell \leq N\right\}$ be the unique adapted solution of (3.3). Denote the drift term in (3.3) by $R^{\ell}(t)$. By Ito's formula and equations (3.15), (3.3), 
we have (with $t$ suppressed)

$$
\begin{aligned}
\mathbb{E} & {\left[\Delta x(T)^{T} h_{x x}^{\ell}(\bar{x}(T)) \Delta x(T)\right]=\mathbb{E}\left[\Delta x(T)^{T} P^{\ell}(T) \Delta x(T)\right] } \\
= & \mathbb{E} \int_{0}^{T}\left\{(\Delta x)^{T} R^{\ell} \Delta x+2(\Delta x)^{T} P^{\ell}\left(\Delta_{x u}^{0} b\right)\right. \\
& \left.+2 \sum_{i=1}^{d}(\Delta x)^{T}\left(Q_{i}^{\ell}\right)\left(\Delta_{x u}^{0} \sigma^{i}\right)+\sum_{i=1}^{d}\left(\Delta_{x u}^{0} \sigma^{i}\right)^{T} P^{\ell}\left(\Delta_{x u}^{0} \sigma^{i}\right)\right\} d t \\
= & \mathbb{E} \int_{0}^{T}\left\{(\Delta x)^{T} R^{\ell} \Delta x+2(\Delta x)^{T} P^{\ell}\left(b_{x} \Delta x+\Delta_{x u}^{1} b\right)\right. \\
& \left.+2 \sum_{i=1}^{d}(\Delta x)^{T}\left(Q_{i}^{\ell}\right)\left(\sigma_{x}^{i} \Delta x+\Delta_{x u}^{1} \sigma^{i}\right)+\sum_{i=1}^{d}\left(\Delta_{x u}^{0} \sigma^{i}\right)^{T} P^{\ell} \Delta_{x u}^{0} \sigma^{i}\right\} d t \\
=\mathbb{E} \int_{0}^{T}\left\{(\Delta x)^{T}\left[R^{\ell}+P^{\ell} b_{x}+\left(b_{x}\right)^{T} P^{\ell}+\sum_{i=1}^{d}\left(Q_{i}^{\ell} \sigma_{x}^{i}+\left(\sigma_{x}^{i}\right)^{T} Q_{i}^{\ell}\right)\right] \Delta x\right. & \left.+2(\Delta x)^{T}\left[P^{\ell} \Delta_{x u}^{1} b+\sum_{i=1}^{d} Q_{i}^{\ell} \Delta_{x u}^{1} \sigma^{i}\right]+\sum_{i=1}^{d}\left(\Delta_{x u}^{0} \sigma^{i}\right)^{T} P^{\ell} \Delta_{x u}^{0} \sigma^{i}\right\} d t \\
& \quad \int_{0}^{T}\left\{-(\Delta x)^{T}\left[H_{x x}^{\ell}+\sum_{i=1}^{d}\left(\sigma_{x}^{i}\right)^{T} P^{\ell} \sigma_{x}^{i}\right] \Delta x\right. \\
& \left.+2(\Delta x)^{T}\left[P^{\ell} \Delta_{x u}^{1} b+\sum_{i=1}^{d} Q_{i}^{\ell} \Delta_{x u}^{1} \sigma^{i}\right]+\sum_{i=1}^{d}\left(\Delta_{x u}^{0} \sigma^{i}\right)^{T} P^{\ell} \Delta_{x u}^{0} \sigma^{i}\right\} d t .
\end{aligned}
$$

Hence,

(3.19)

$$
\begin{aligned}
& J^{\ell}\left(u_{\varepsilon}(\cdot)\right)-J^{\ell}(\bar{u}(\cdot))=\mathbb{E}\left[\Delta_{x}^{1} h^{\ell}(T)+\int_{0}^{T} \Delta_{x u}^{1} H^{\ell}(t) d t\right] \\
& =\mathbb{E}\left\{\Delta_{x}^{2} h^{\ell}(T)+\frac{1}{2} \Delta x(T)^{T} h_{x x}^{\ell}(\bar{x}(T)) \Delta x(T)\right. \\
& \left.\quad+\int_{0}^{T}\left[\Delta_{x u}^{2} H^{\ell}(t)+\frac{1}{2} \Delta x(t)^{T} H_{x x}^{\ell}(t) \Delta x(t)\right] d t\right\} \\
& =\mathbb{E}\left\{\Delta_{x}^{2} h^{\ell}(T)+\int_{0}^{T}\left[\Delta_{x u}^{2} H^{\ell}(t)+\Delta x(t)^{T}\left(P^{\ell}(t) \Delta_{x u}^{1} b(t)+\sum_{i=1}^{d} Q_{i}^{\ell}(t) \Delta_{x u}^{1} \sigma^{i}(t)\right)\right.\right. \\
& \left.\quad+\frac{1}{2} \sum_{i=1}^{d}\left(\Delta_{x u}^{0} \sigma^{i}(t)^{T} P^{\ell}(t) \Delta_{x u}^{0} \sigma^{i}(t)-\Delta x(t)^{T} \sigma_{x}^{i}(t)^{T} P^{\ell}(t) \sigma_{x}^{i}(t) \Delta x(t)\right] d t\right\} .
\end{aligned}
$$

This proves the second equality in (3.13). 


\section{A Variational Formula for the Performance Functional Vector}

In this section, we will obtain a directional derivative for $J(\cdot)$ at a given admissible control process $\bar{u}(\cdot) \in \mathcal{U}^{\beta}[0, T]$ in any given "control process direction". We will make this clear shortly. Since $U$ is not necessarily convex, one has to consider spike variations of $\bar{u}(\cdot)$. Let

$$
\mathcal{G}_{\varepsilon} \triangleq\{G \subseteq[0, T]|| G \mid=\varepsilon T\},
$$

where $|G|$ stands for the Lebesgue measure of $G$. Let $v(\cdot) \in \mathcal{U}^{\beta}[0, T]$. Let $G_{\varepsilon} \in \mathcal{G}_{\varepsilon}$ for $\varepsilon \in(0,1)$. Consider the variation

$$
u_{\varepsilon}(t) \triangleq \begin{cases}v(t), & t \in G_{\varepsilon}, \\ \bar{u}(t), & \text { otherwise. }\end{cases}
$$

Let $\bar{x}(\cdot)$ and $x_{\varepsilon}(\cdot)$ be the state processes with $\bar{x}(0)=x_{\varepsilon}(0) \in \mathbb{R}^{n}$ corresponding to the controls $\bar{u}(\cdot)$ and $u_{\varepsilon}(\cdot)$. Let $\left\{\left(p^{\ell}(\cdot), q^{\ell}(\cdot)\right), 1 \leq \ell \leq N\right\}$ and $\left\{\left(P^{\ell}, Q^{\ell}\right), 1 \leq \ell \leq N\right\}$ be the first order and second order adjoint processes associated with $\bar{u}(\cdot)$, determined by (3.2) and (3.3), respectively. Define for $(t, u) \in[0, T] \times U$,

$$
\left\{\begin{array}{l}
\mathcal{H}^{\ell}(t, u)=\Delta_{u} H^{\ell}(t, u)+\frac{1}{2} \sum_{i=1}^{d} \Delta_{u} \sigma^{i}(t, u)^{T} P^{\ell}(t) \Delta_{u} \sigma^{i}(t, u), \\
\Delta_{u} H^{\ell}(t, u)=H^{\ell}\left(t, \bar{x}(t), u, p^{\ell}(t), q^{\ell}(t)\right)-H^{\ell}\left(t, \bar{x}(t), \bar{u}(t), p^{\ell}(t), q^{\ell}(t)\right), \\
\Delta_{u} \sigma^{i}(t, u)=\sigma^{i}(t, \bar{x}(t), u)-\sigma^{i}(t, \bar{x}(t), \bar{u}(t)) .
\end{array}\right.
$$

Compare the notations here with those in (3.11) and (3.12).

Theorem 4.1. Let $(S 1)-(S 2)$ hold and $\beta>3$. Let $(\bar{x}(\cdot), \bar{u}(\cdot)) \in L_{\mathcal{F}}^{\beta}\left(\Omega, C\left([0, T], \mathbb{R}^{n}\right)\right) \times$ $\mathcal{U}^{\beta}[0, T]$ and $v(\cdot) \in \mathcal{U}^{\beta}[0, T]$ be given above. Then there exists a family $\left\{G_{\varepsilon} \in \mathcal{G}_{\varepsilon}\right\}$ for $\varepsilon \in(0,1)$ such that the variation $u_{\varepsilon}(\cdot)$ defined in (4.2) satisfies

$$
\begin{aligned}
\left.\frac{d}{d \varepsilon} J^{\ell}\left(u_{\varepsilon}(\cdot)\right)\right|_{\varepsilon=0} & =\lim _{\varepsilon \rightarrow 0+} \frac{1}{\varepsilon} \mathbb{E} \int_{G_{\varepsilon}} \mathcal{H}^{\ell}(t, v(t)) \\
& =\mathbb{E} \int_{0}^{T} \mathcal{H}^{\ell}(t, v(t)) d t .
\end{aligned}
$$

Recall that under (S1)-(S2), if $U$ is convex, and all the maps involved are also differentiable in $u$, then by usual convex perturbation, one can show that the map $u(\cdot) \mapsto J^{\ell}(u(\cdot))$ is Gâteaux differentiable, and the directional derivative in the direction of $v(\cdot)$ is linear in $v(\cdot)$; see [2] and [4]. Now by making spike perturbation, which is applicable for general $U$, we obtain the above "directional derivative" which is not linear in the "direction" $v(\cdot)$. Hence, the above "directional derivative" is significantly different from Gâteaux directional derivative. 
A similar result was proved in [12] under the assumption that

$$
\sup _{0 \leq t \leq T} \mathbb{E}|\bar{u}(t)|^{k}<\infty, \quad \forall k \geq 1 .
$$

Ideally, one should be able to prove $(4.4)$ for $\bar{u}(\cdot) \in \mathcal{U}^{2}[0, T]$ because the optimal control is well-posed in this space.

The proof of Theorem 4.1 is divided into two parts. In Part I, we construct $G_{\varepsilon} \in \mathcal{G}_{\varepsilon}$ that satisfy the second equality in (4.4). Then in Part II, we show that the resulting spike variation $u_{\varepsilon}(\cdot)$ satisfies the first equality in (4.4) as well.

For Part I of the proof, we need the following lemma.

Lemma 4.1. Let $\alpha_{i}, \beta_{i} \in[1, \infty), \ell_{i} \geq 1, i=1,2, \cdots, k$, and $\varepsilon \in(0,1)$. Then for any $\psi_{i}(\cdot) \in L_{\mathcal{F}}^{\beta_{i}}\left(\Omega ; L^{\alpha_{i}}\left(0, T ; \mathbb{R}^{\ell_{i}}\right)\right), i=1,2, \cdots, k$,

$$
\inf _{G \in \mathcal{G}_{\varepsilon}} \max _{1 \leq i \leq k} \mathbb{E}\left|\int_{0}^{T}\left(1-\frac{I_{G}(t)}{\varepsilon}\right) \psi_{i}(t) d t\right|^{\beta_{i}}=0,
$$

where $I_{G}(\cdot)$ is the characteristic function of $G$.

The proof of the above result is essentially the same as a result found in [9]. For readers' convenience, we present a proof here.

Proof of Lemma 4.1. Since $\psi_{i}(\cdot) \in L_{\mathcal{F}}^{\beta_{i}}\left(\Omega ; L^{\alpha_{i}}\left(0, T ; \mathbb{R}^{\ell_{i}}\right)\right)$, for $\delta=\varepsilon^{\beta_{i}+1}$, there exists a process $\psi_{i}^{\delta}(\cdot)$ of form

$$
\psi_{i}^{\delta}(t)=\sum_{j=0}^{n} \xi_{i}^{j} I_{\left[t_{j}, t_{j+1}\right]}(t), \quad 1 \leq i \leq k,
$$

with $0=t_{0}<t_{1}<\cdots<t_{n+1}=T$, $\xi_{j}^{i}$ being $\mathcal{F}_{t_{j}}$-measurable, such that

$$
\sum_{i=1}^{k} \mathbb{E}\left(\int_{0}^{T}\left|\psi_{i}(t)-\psi_{i}^{\delta}(t)\right|^{\alpha_{i}} d t\right)^{\frac{\beta_{i}}{\alpha_{i}}}<\delta
$$

Note that we can always choose the partition $\left\{t_{j}\right\}_{0 \leq j \leq n+1}$ independent of $i=1, \cdots, k$. Now we let

$$
G=\bigcup_{j=0}^{n}\left[t_{j}, t_{j}+\varepsilon\left(t_{j+1}-t_{j}\right)\right] .
$$

Then $|G|=\varepsilon T$. Thus, $G \in \mathcal{G}_{\varepsilon}$, and

$$
\int_{0}^{T}\left(1-\frac{I_{G}(t)}{\varepsilon}\right) \psi_{i}^{\delta}(t) d t=\sum_{j=1}^{n} \xi_{i}^{j}\left[\left(t_{j+1}-t_{j}\right)-\frac{\varepsilon\left(t_{j+1}-t_{j}\right)}{\varepsilon}\right]=0, \quad \text { a.s. }
$$


Hence, for each $1 \leq i \leq k$,

$$
\begin{aligned}
& \mathbb{E}\left|\int_{0}^{T}\left(1-\frac{I_{G}(t)}{\varepsilon}\right) \psi_{i}(t) d t\right|^{\beta_{i}} \\
& =\mathbb{E}\left|\int_{0}^{T}\left(1-\frac{I_{G}(t)}{\varepsilon}\right)\left[\psi_{i}(t)-\psi_{i}^{\delta}(t)\right] d t\right|^{\beta_{i}} \\
& \leq\left(1+\frac{1}{\varepsilon}\right)^{\beta_{i}} T^{\frac{\left(\alpha_{i}-1\right) \beta_{i}}{\alpha_{i}}} \mathbb{E}\left(\int_{0}^{T}\left|\psi_{i}(t)-\psi_{i}^{\delta}(t)\right|^{\alpha_{i}} d t\right)^{\frac{\beta_{i}}{\alpha_{i}}} \\
& <\left(1+\frac{1}{\varepsilon}\right)^{\beta_{i}} T^{\frac{\left(\alpha_{i}-1\right) \beta_{i}}{\alpha_{i}}} \delta=(1+\varepsilon)^{\beta_{i}} T^{\frac{\left(\alpha_{i}-1\right) \beta_{i}}{\alpha_{i}}} \varepsilon .
\end{aligned}
$$

By letting $\varepsilon \rightarrow 0+$, we obtain (4.6).

Proof of Theorem 4.1 (Part I). In this part we construct $G_{\varepsilon} \in \mathcal{G}_{\varepsilon}$ for $\varepsilon \in(0,1)$ that satisfies the second equality of (4.4). Denote by $\psi_{0}(\cdot)$ and $\eta(\cdot)$ the following processes.

$$
\begin{aligned}
& \psi_{0}(t)=2 \rho(t)+2|\bar{x}(t)|+|\bar{u}(t)|+|v(t)|, \\
& \eta(t)=1+\sum_{\ell=1}^{N}\left(\left|p^{\ell}(t)\right|+\left|q^{\ell}(t)\right|+\left|P^{\ell}(t)\right|+\left|Q^{\ell}(t)\right|\right),
\end{aligned}
$$

where $\rho$ appears in assumption (S2). We claim

$$
\begin{aligned}
& \psi_{0}(\cdot), \eta(\cdot), \Delta_{u} \sigma^{i}(\cdot, v(\cdot)) \in L_{\mathcal{F}}^{\beta}\left(\Omega ; L^{2}(0, T ; \mathbb{R})\right), \\
& \mathcal{H}(\cdot, v(\cdot)) \in L_{\mathcal{F}}^{1}\left(\Omega ; L^{1}(0, T ; \mathbb{R})\right) .
\end{aligned}
$$

Indeed, by $(2.3)$ or $(2.11)$, we know that $\|\bar{x}(\cdot)\|_{\infty, \beta} \leq C$. So Proposition 3.1 and (4.12) imply that $\psi_{0}(\cdot), \eta(\cdot) \in L_{\mathcal{F}}^{\beta}\left(\Omega ; L^{2}(0, T ; \mathbb{R})\right)$. By assumption $(\mathrm{S} 2)$, for $\varphi=b$ and $\sigma$

$$
\begin{aligned}
& \left|\Delta_{u} \varphi(t, v(t))\right|=|\varphi(t, \bar{x}(t), v(t))-\varphi(t, \bar{x}(t), \bar{u}(t))| \leq \psi_{0}(t) \\
& \left|\Delta_{u} H^{\ell}(t, v(t))\right| \leq L\left(\psi_{0}(t)+\left|p^{\ell}(t)\right|+\left|q^{\ell}(t)\right|\right) \psi_{0}(t) .
\end{aligned}
$$

It follows that

$$
\begin{aligned}
& \left|\mathcal{H}^{\ell}(t, v(t))\right| \leq C\left(\psi_{0}(t)^{2}+|\eta(t)|^{2}\right)+\frac{1}{2} \sum_{i=1}^{d}\left|\Delta_{u} \sigma^{i}(t, v(t))\right|^{2}\left|P^{\ell}(t)\right| \\
& \leq C\left[\psi_{0}(t)^{2}+\eta(t)^{2}+\left|P^{\ell}(t)\right| \psi_{0}(t)^{2}\right] .
\end{aligned}
$$

Since $\beta>3$, by (3.6) in Proposition 3.1 and the fact $\psi_{0}(\cdot) \in L_{\mathcal{F}}^{\beta}\left(\Omega ; L^{2}(0, T ; \mathbb{R})\right)$, we obtain

$$
\begin{aligned}
& \mathbb{E} \int_{0}^{T}\left|\psi_{0}(t)\right|^{2}\left|P^{\ell}(t)\right| d t \leq \mathbb{E} \sup _{t \in[0, T]}\left|P^{\ell}(t)\right| \int_{0}^{T}\left|\psi_{0}(t)\right|^{2} d t \\
& \leq\left[\mathbb{E} \sup _{t \in[0, T]}\left|P^{\ell}(t)\right|^{\beta}\right]^{\frac{1}{\beta}}\left[\mathbb{E}\left(\int_{0}^{T}\left|\psi_{0}(t)\right|^{2} d t\right)^{\frac{\beta}{2}}\right]^{\frac{2}{\beta}}<\infty .
\end{aligned}
$$


This, combined with (4.15), implies that $\mathcal{H}(\cdot, v(\cdot)) \in L_{\mathcal{F}}^{1}\left(\Omega ; L^{1}(0, T ; \mathbb{R})\right)$.

By Lemma 4.1 (or its proof) applied to $\mathcal{H}^{\ell}(\cdot, v(\cdot))$ and $\psi_{0}(\cdot)$, we obtain the existence of $G_{\varepsilon} \in \mathcal{G}_{\varepsilon}$ for $\varepsilon \in(0,1)$ such that

$$
\mathbb{E} \int_{G_{\varepsilon}} \mathcal{H}^{\ell}(t, v(t)) d t=\varepsilon \int_{0}^{T} \mathcal{H}^{\ell}(t, v(t)) d t+o(\varepsilon),
$$

$$
\mathbb{E}\left(\int_{G_{\varepsilon}} \psi_{0}(t)^{2} d t\right)^{\frac{\beta}{2}} \leq C \varepsilon^{\frac{\beta}{2}}
$$

where $C=\mathbb{E}\left(\int_{0}^{T} \psi_{0}(t)^{2} d t\right)^{\frac{\beta}{2}}+1$. Clearly, (4.17) implies the second equality of (4.4) for $G_{\varepsilon}$.

Now we prepare to go Part II of the proof of Theorem 4.1. Let $\psi_{0}$ and $\eta$ be defined in (4.12). For $\varepsilon>0$, define

$$
\psi_{\varepsilon}(t)=2 \rho(t)+|\bar{x}(t)|+\left|x_{\varepsilon}(t)\right|+|\bar{u}(t)|+\left|u_{\varepsilon}(t)\right| .
$$

We will see that $\psi_{\varepsilon}(\cdot)$ appears many times in the proof of Theorem 4.1, Part II. We need the following technical lemma.

Lemma 4.2. Let the assumptions of Theorem 4.1 hold. Choose $G_{\varepsilon}$ to satisfy (4.17) and (4.18), and let $I_{\varepsilon}(t)$ be the characteristic function of $G_{\varepsilon}$. Let $x_{\varepsilon}(\cdot)$ be the state process with $x_{\varepsilon}(0)=\bar{x}(0)$ corresponding to $u_{\varepsilon}(\cdot)$ defined in (4.2). Let $\Delta x(\cdot)=x_{\varepsilon}(\cdot)-\bar{x}(\cdot)$. Then for $\alpha \in(0, \beta]$ and $\gamma>0$,

$$
\begin{gathered}
\mathbb{E}\left[\sup _{t \in[0, T]}|\Delta x(t)|^{\alpha}\right] \leq C \varepsilon^{\frac{\alpha}{2}} . \\
\mathbb{E}\left[\sup _{t \in[0, T]} \tilde{\omega}(|\Delta x(t)|)^{\gamma}\right]=o(1) .
\end{gathered}
$$

Moreover, for $\lambda=0,1$ and $\theta_{1}, \theta_{2}, \theta_{3}, \theta_{4}$ with

$$
\begin{aligned}
& 0 \leq \theta_{1}, \theta_{2}, \theta_{3}, \theta_{4} ; \quad \theta_{1}, \theta_{2}, \theta_{1}+\theta_{2} \leq 2, \\
& \theta_{1} \theta_{4}, \theta_{2} \theta_{4}, \theta_{3} \theta_{4},\left(\theta_{1}+\theta_{2}+\theta_{3}\right) \theta_{4} \leq \beta,
\end{aligned}
$$

the following holds.

$$
\mathbb{E}\left(\int_{0}^{T} \psi_{\varepsilon}(t)^{\theta_{1}} \eta(t)^{\theta_{2}}|\Delta x(t)|^{\theta_{3}} I_{\varepsilon}^{\lambda}(t) d t\right)^{\theta_{4}}=o(1)^{\lambda \theta_{2} \theta_{4}}\left[\lambda\left(1-\frac{\theta_{2}}{2}\right)+\frac{\theta_{3}}{2}\right] \theta_{4},
$$

where $o(1)^{\lambda \theta_{2} \theta_{4}}=o(1)$ if $\lambda \theta_{2} \theta_{4}>0$, and by convention, $o(1)^{0}=1$. 
Proof. By (4.18), $\left\|\psi_{0} I_{\varepsilon}\right\|_{2, \beta} \leq C \varepsilon^{\frac{1}{2}}$. Then by (2.11) with $\Delta x(0)=0$ and $u(\cdot)=u_{\varepsilon}(\cdot)$, we have

$$
\|\Delta x(\cdot)\|_{\infty, \beta} \leq C||(2|\bar{x}|+2 \rho+|\bar{u}|+|v|) I_{\varepsilon}\left\|_{2, \beta} \leq C|| \psi_{0} I_{\varepsilon}\right\|_{2, \beta} \leq C \varepsilon^{\frac{1}{2}} .
$$

This shows (4.19) for $\alpha=\beta$. For $\alpha \in(0, \beta]$, we have that

$$
\mathbb{E}\left[\sup _{t \in[0, T]}|\Delta x(t)|^{\alpha}\right] \leq\left[\mathbb{E}\left(\sup _{t \in[0, T]}|\Delta x(t)|^{\beta}\right)\right]^{\frac{\alpha}{\beta}} \leq C \varepsilon^{\frac{\alpha}{2}} .
$$

For (4.20), we may assume that $\gamma=1$ since $\tilde{\omega}(\cdot)^{\gamma}$ is just another modulus of continuity. For each $\delta>0$, there exists a constant $K_{\delta}>0$ such that $\tilde{\omega}(r) \leq \delta+r^{\beta} K_{\delta}$ for all $r \geq 0$. It follows that

$$
\mathbb{E} \sup _{0 \leq t \leq T} \tilde{\omega}(|\Delta x(t)|) \leq \mathbb{E} \sup _{0 \leq t \leq T}\left\{\delta+K_{\delta}|\Delta x(t)|^{\beta}\right\} \leq \delta+C K_{\delta} \varepsilon^{\frac{\beta}{2}} .
$$

Choosing $\delta$ small enough and then letting $\varepsilon$ be sufficiently small, we obtain (4.20).

Now we prove (4.22). Note that if $\theta_{1}, \theta_{2}, \theta_{3}, \theta_{4}$ satisfy all of the strict inequalities in (4.21), then by Hölder's inequality we have (with $t$ suppressed)

$$
\begin{aligned}
& \mathbb{E}\left(\int_{0}^{T} \psi_{\varepsilon}^{\theta_{1}} \eta^{\theta_{2}}|\Delta x|^{\theta_{3}} I_{\varepsilon}^{\lambda} d t\right)^{\theta_{4}} \leq \mathbb{E}\left(\sup _{t \in[0, T]}|\Delta x|^{\theta_{3}} \int_{0}^{T} \psi_{\varepsilon}^{\theta_{1}} \eta^{\theta_{2}} I_{\varepsilon}^{\lambda} d t\right)^{\theta_{4}} \\
& \leq \mathbb{E}\left[\left(\sup _{t \in[0, T]}|\Delta x|^{\theta_{3} \theta_{4}}\right)\left(\int_{0}^{T} \psi_{\varepsilon}^{2} I_{\varepsilon}^{\lambda} d t\right)^{\frac{\theta_{1} \theta_{4}}{2}}\left(\int_{0}^{T} \eta^{2} I_{\varepsilon}^{\lambda} d t\right)^{\frac{\theta_{2} \theta_{4}}{2}}\left(\int_{0}^{T} I_{\varepsilon}^{\lambda} d t\right)^{\frac{2-\theta_{1}-\theta_{2}}{2} \theta_{4}}\right] \\
& \leq \varepsilon^{\frac{\lambda\left(2-\theta_{1}-\theta_{2}\right) \theta_{4}}{2}}\|\Delta x\|_{\infty, \beta}^{\theta_{3} \theta_{4}}\left\|\psi_{\varepsilon} I_{\varepsilon}^{\lambda}\right\|_{2, \beta}^{\theta_{1} \theta_{4}}\left\|\eta I_{\varepsilon}^{\lambda}\right\|_{2, \beta}^{\theta_{2} \theta_{4}} .
\end{aligned}
$$

However, with the convention $(\cdot)^{0}=1$, (4.23) holds for all $\theta_{1}, \theta_{2}, \theta_{3}, \theta_{4}$ in (4.21). Now we estimate the terms in (4.23). By (2.11) and (4.19), $\left\|x_{\varepsilon}\right\|_{\infty, \beta} \leq C$. By (4.18) again, $\left\|\psi_{0} I_{\varepsilon}\right\|_{2, \beta} \leq C \varepsilon^{\frac{1}{2}}$. Thus

$$
\begin{aligned}
\left\|\psi_{\varepsilon} I_{\varepsilon}\right\|_{2, \beta} & =\left\|\left(2 \rho+|\bar{x}|+\left|x_{\varepsilon}\right|+|\bar{u}|+|v|\right) I_{\varepsilon}\right\|_{2, \beta} \\
& \leq\left\|\left|x_{\varepsilon} I_{\varepsilon}\left\|\left.\right|_{2, \beta}+\right\| \psi_{0} I_{\varepsilon} \|_{2, \beta}\right.\right. \\
& \leq\left\|x_{\varepsilon}\right\|_{\infty, \beta}\left|G_{\varepsilon}\right|^{\frac{1}{2}}+C \varepsilon^{\frac{1}{2}} \leq C_{1} \varepsilon^{\frac{1}{2}} \\
\left\|\eta I_{\varepsilon}\right\|_{2, \beta} & =o(1) \\
\left\|\psi_{\varepsilon}+\eta\right\|_{2, \beta} & \leq C .
\end{aligned}
$$

The inequalities in (4.24) can be combined as, for $\lambda=0$ or 1 ,

$$
\left\|\psi_{\varepsilon} I_{\varepsilon}^{\lambda}\right\|_{2, \beta} \leq C \varepsilon^{\frac{\lambda}{2}} ; \quad\left\|\eta I_{\varepsilon}^{\lambda}\right\|_{2, \beta}=o(1)^{\lambda} .
$$

Substituting (4.25) into (4.23), we obtain (4.22):

$$
\begin{aligned}
& \mathbb{E}\left(\int_{0}^{T} \psi_{\varepsilon}^{\theta_{1}} \eta^{\theta_{2}}|\Delta x|^{\theta_{3}} I_{\varepsilon}^{\lambda} d t\right)^{\theta_{4}} \leq C \varepsilon^{\lambda\left(1-\frac{\theta_{1}+\theta_{2}}{2}\right) \theta_{4}+\frac{\theta_{3}}{2} \theta_{4}+\lambda \frac{\theta_{1}}{2} \theta_{4}} o(1)^{\lambda \theta_{2} \theta_{4}} \\
& =o(1)^{\lambda \theta_{2} \theta_{4}} \varepsilon\left[\lambda\left(1-\frac{\theta_{2}}{2}\right)+\frac{\theta_{3}}{2}\right] \theta_{4} .
\end{aligned}
$$


We are ready for Part II of the proof of Theorem 4.1.

Proof of Theorem 4.1 (Part II). For convenience, we rewrite (3.13) below (4.27)

$$
\begin{aligned}
& J^{\ell}\left(u_{\varepsilon}(\cdot)\right)-J^{\ell}(\bar{u}(\cdot)) \\
& =\mathbb{E}\left\{\Delta_{x}^{2} h^{\ell}(T)+\int_{0}^{T}\left[\Delta_{x u}^{2} H^{\ell}(t)+\Delta x(t)^{T}\left(P^{\ell}(t) \Delta_{x u}^{1} b(t)+\sum_{i=1}^{d} Q_{i}^{\ell}(t) \Delta_{x u}^{1} \sigma^{i}(t)\right)\right.\right. \\
& \left.\left.\quad+\frac{1}{2} \sum_{i=1}^{d}\left(\Delta_{x u}^{0} \sigma^{i}(t)^{T} P^{\ell}(t) \Delta_{x u}^{0} \sigma^{i}(t)-\Delta x(t)^{T} \sigma_{x}^{i}(t)^{T} P^{\ell}(t) \sigma_{x}^{i}(t) \Delta x(t)\right)\right] d t\right\} .
\end{aligned}
$$

We list the following claims:

$$
\begin{gathered}
\mathbb{E}\left[\Delta_{x}^{2} h^{\ell}(T)\right]=o(\varepsilon) . \\
\mathbb{E} \int_{0}^{T} \Delta_{x u}^{2} H^{\ell}(t) d t=\mathbb{E} \int_{0}^{T} \Delta_{u} H^{\ell}\left(t, u_{\varepsilon}(t)\right) d t+o(\varepsilon) . \\
\mathbb{E} \int_{0}^{T} \Delta x(t)^{T}\left[P^{\ell}(t) \Delta_{x u}^{1} b(t)+\sum_{i=1}^{d} Q_{i}^{\ell}(t) \Delta_{x u}^{1} \sigma^{i}(t)\right] d t=o(\varepsilon) . \\
\mathbb{E} \int_{0}^{T}\left(\Delta_{x u}^{0} \sigma^{i}(t)^{T} P^{\ell}(t) \Delta_{x u}^{0} \sigma^{i}(t)-\Delta x(t)^{T} \sigma_{x}^{i}(t)^{T} P^{\ell}(t) \sigma_{x}^{i}(t) \Delta x(t)\right) d t \\
=\mathbb{E} \int_{0}^{T} \Delta_{u} \sigma^{i}\left(t, u_{\varepsilon}(t)\right)^{T} P^{\ell}(t) \Delta_{u} \sigma^{i}\left(t, u_{\varepsilon}(t)\right) d t+o(\varepsilon) .
\end{gathered}
$$

If the above are all proved, then (4.27) becomes

$$
\begin{aligned}
J^{\ell}\left(u_{\varepsilon}(\cdot)\right)-J(\bar{u}(\cdot)) & =\mathbb{E} \int_{0}^{T}\left[\Delta_{u} H^{\ell}\left(t, u_{\varepsilon}(t)\right)\right. \\
& \left.+\frac{1}{2} \sum_{i=1}^{d} \Delta_{u} \sigma^{i}\left(t, u_{\varepsilon}(t)\right) P^{\ell}(t) \Delta_{u} \sigma^{i}\left(t, u_{\varepsilon}(t)\right)\right] d t+o(\varepsilon) \\
& =\mathbb{E} \int_{0}^{T} \mathcal{H}^{\ell}\left(t, u_{\varepsilon}(t)\right) d t+o(\varepsilon) \\
& =\mathbb{E} \int_{G_{\varepsilon}} \mathcal{H}^{\ell}(t, v(t)) d t+o(\varepsilon) .
\end{aligned}
$$

Here we used the fact that $\mathcal{H}^{\ell}\left(t, u_{\varepsilon}(t)\right)=\mathcal{H}^{\ell}(t, v(t)) I_{\varepsilon}(t)$. This implies the first equality in (4.4).

We now prove (4.28)-(4.31). 
Proof of (4.28): We note that

$$
\begin{aligned}
& \Delta_{x}^{2} h^{\ell}(T)=h^{\ell}\left(x_{\varepsilon}(T)\right)-h^{\ell}(\bar{x}(T))-h_{x}^{\ell}(\bar{x}(T))^{T} \Delta x(T)-\frac{1}{2} \Delta x(T)^{T} h_{x x}^{\ell}(\bar{x}(T)) \Delta x(T) \\
& =\int_{0}^{1}\left[h_{x}^{\ell}(\bar{x}(T)+\lambda \Delta x(T))-h_{x}^{\ell}(\bar{x}(T))\right]^{T} \Delta x(T) d \lambda-\frac{1}{2} \Delta x(T)^{T} h_{x x}^{\ell}(\bar{x}(T)) \Delta x(T) \\
& =\int_{0}^{1} \int_{0}^{1} \Delta x(T)^{T} h_{x x}^{\ell}(\bar{x}(T)+\lambda \mu \Delta x(T)) \Delta x(T) d \mu d \lambda-\frac{1}{2} \Delta x(T)^{T} h_{x x}^{\ell}(\bar{x}(T)) \Delta x(T) \\
& =\Delta x(T)^{T}\left[\int_{0}^{1} \lambda\left(h_{x x}^{\ell}\left(\lambda \bar{x}(T)+(1-\lambda) x_{\varepsilon}(T)\right)-h_{x x}^{\ell}(\bar{x}(T))\right) d \lambda\right] \Delta x(T) .
\end{aligned}
$$

Hence, by assumption (S2), (4.19), and (4.20) with $\gamma=\frac{\beta}{\beta-2}$ to obtain

$$
\begin{aligned}
& \left|\mathbb{E} \Delta_{x}^{2} h^{\ell}(T)\right| \\
& \leq \mathbb{E}\left(\widetilde{\omega}(\mid \Delta x(T))|\Delta x(T)|^{2}\right) \leq\left\{\mathbb{E}\left[\widetilde{\omega}(|\Delta x(T)|]^{\frac{\beta}{\beta-2}}\right\}^{\frac{\beta-2}{\beta}}\left[\mathbb{E}|\Delta x(T)|^{\beta}\right]^{\frac{2}{\beta}}\right. \\
& \leq C \varepsilon\left\{\mathbb{E}\left[\widetilde{\omega}(|\Delta x(T)|]^{\frac{\beta}{\beta-2}}\right\}^{\frac{\beta-2}{\beta}}=o(\varepsilon),\right.
\end{aligned}
$$

which proves (4.28).

Proof of (4.29): Recalling the definitions (3.12) and (4.3) of $\Delta_{x u}^{2} H(t)$ and $\Delta_{u} H\left(t, u_{\varepsilon}(t)\right)$, we have (suppressing $t$ and $\left(p^{\ell}, q^{\ell}\right)$ when it is clear)

$$
\begin{aligned}
& \left|\Delta_{x u}^{2} H^{\ell}(t)-\Delta_{u} H^{\ell}\left(t, u_{\varepsilon}(t)\right)\right| \\
& =\mid H^{\ell}\left(x_{\varepsilon}, u_{\varepsilon}\right)-H^{\ell}(\bar{x}, \bar{u})-H_{x}^{\ell}(\bar{x}, \bar{u})^{T} \Delta x-\frac{1}{2}(\Delta x)^{T} H_{x x}^{\ell}(\bar{x}, \bar{u}) \Delta x \\
& -H^{\ell}\left(\bar{x}, u_{\varepsilon}\right)+H^{\ell}(\bar{x}, \bar{u}) \mid \\
& =\left|\left[\int_{0}^{1} H_{x}^{\ell}\left(\bar{x}+\lambda \Delta x, u_{\varepsilon}\right) d \lambda-H_{x}^{\ell}(\bar{x}, \bar{u})\right]^{T} \Delta x-\frac{1}{2}(\Delta x)^{T} H_{x x}^{\ell}(\bar{x}, \bar{u}) \Delta x\right| \\
& =\mid\left[\int_{0}^{1}\left(H_{x}^{\ell}\left(\bar{x}+\lambda \Delta x, u_{\varepsilon}\right)-H_{x}^{\ell}(\bar{x}+\lambda \Delta x, \bar{u})\right) d \lambda\right]^{T} \Delta x \\
& +\left[\int_{0}^{1}\left(H_{x}^{\ell}(\bar{x}+\lambda \Delta x, \bar{u})-H_{x}^{\ell}(\bar{x}, \bar{u})\right) d \lambda\right]^{T} \Delta x-\frac{1}{2}(\Delta x)^{T} H_{x x}^{\ell}(\bar{x}, \bar{u}) \Delta x \mid \\
& =\mid\left[\int_{0}^{1}\left(H_{x}^{\ell}\left(\bar{x}+\lambda \Delta x, u_{\varepsilon}\right)-H_{x}^{\ell}(\bar{x}+\lambda \Delta x, \bar{u})\right) d \lambda\right]^{T} \Delta x \\
& +(\Delta x)^{T}\left[\int_{0}^{1} \lambda\left(H_{x x}^{\ell}\left(\lambda \bar{x}+(1-\lambda) x_{\varepsilon}, \bar{u}\right)-H_{x x}^{\ell}(\bar{x}, \bar{u})\right) d \lambda\right]^{T} \Delta x \mid .
\end{aligned}
$$

Since

$$
\begin{aligned}
& \left|H_{x x}^{\ell}\left(t, \lambda \bar{x}(t)+(1-\lambda) x_{\varepsilon}(t), \bar{u}(t), p^{\ell}(t), q^{\ell}(t)\right)-H_{x x}^{\ell}\left(t, \bar{x}(t), \bar{u}(t), p^{\ell}(t), q^{\ell}(t)\right)\right| \\
& \leq\left[1+\left|p^{\ell}(t)\right|+\left|q^{\ell}(t)\right|\right] \widetilde{\omega}(|\Delta x(t)|) \leq \eta(t) \widetilde{\omega}(|\Delta x(t)|),
\end{aligned}
$$


by (4.20) with $\gamma=\frac{\beta}{\beta-3}$ and (4.22) with $\left(\theta_{1}, \theta_{2}, \theta_{3}, \theta_{4}, \lambda\right)=\left(0,1,2, \frac{\beta}{3}, 0\right)$, we have (note $\beta>3)$

$$
\begin{aligned}
& \mathbb{E} \int_{0}^{T}\left|(\Delta x)^{T}\left[\int_{0}^{1} \lambda\left(H_{x x}^{\ell}\left(\lambda \bar{x}+(1-\lambda) x_{\varepsilon}, \bar{u}\right)-H_{x x}^{\ell}(\bar{x}, \bar{u})\right) d \lambda\right]^{T} \Delta x\right| d t \\
& \leq \mathbb{E} \int_{0}^{T} \eta(t) \widetilde{\omega}(|\Delta x(t)|)|\Delta x(t)|^{2} d t \leq \mathbb{E}\left[\sup _{t \in[0, T]} \widetilde{\omega}(|\Delta x(t)|) \int_{0}^{T} \eta(t)|\Delta x(t)|^{2} d t\right] \\
& \leq \mathbb{E}\left[\sup _{t \in[0, T]} \widetilde{\omega}(|\Delta x(t)|)^{\frac{\beta}{\beta-3}}\right]^{\frac{\beta-3}{\beta}}\left(\mathbb{E}\left[\int_{0}^{T} \eta(t)|\Delta x(t)|^{2} d t\right]^{\frac{\beta}{3}}\right)^{\frac{3}{\beta}} \\
& =o(1)\left(\varepsilon^{\frac{\beta}{3}}\right)^{\frac{3}{\beta}}=o(\varepsilon) .
\end{aligned}
$$

Also, by (4.22) with $\left(\theta_{1}, \theta_{2}, \theta_{3}, \theta_{4}, \lambda\right)=(1,0,1,1,1)$ and $(0,1,1,1,1)$, respectively,

$$
\begin{aligned}
& \mathbb{E} \int_{0}^{T}\left|\left[\int_{0}^{1}\left(H_{x}^{\ell}\left(\bar{x}+\lambda \Delta x, u_{\varepsilon}\right)-H_{x}^{\ell}(\bar{x}+\lambda \Delta x, \bar{u})\right) d \lambda\right]^{T} \Delta x\right| d t \\
& \leq C \mathbb{E} \int_{0}^{T}\left[\psi_{\varepsilon}(t)+\eta(t)\right]|\Delta x(t)| I_{\varepsilon}(t) d t \leq C\left[\varepsilon^{\frac{3}{2}}+o(1) \varepsilon\right]=o(\varepsilon) .
\end{aligned}
$$

Combining (4.35)-(4.38), we obtain (4.29).

Proof of (4.30): In fact, we have (suppressing $t$ )

$$
\begin{aligned}
& \left|\Delta_{x u}^{1} b(t)\right| \equiv\left|b\left(x_{\varepsilon}, u_{\varepsilon}\right)-b(\bar{x}, \bar{u})-b_{x}(\bar{x}, \bar{u}) \Delta x\right| \\
& =\left|b\left(x_{\varepsilon}, u_{\varepsilon}\right)-b\left(x_{\varepsilon}, \bar{u}\right)+\left[b\left(x_{\varepsilon}, \bar{u}\right)-b(\bar{x}, \bar{u})-b_{x}(\bar{x}, \bar{u}) \Delta x\right]\right| \\
& =\left|\left[b\left(x_{\varepsilon}, u_{\varepsilon}\right)-b\left(x_{\varepsilon}, \bar{u}\right)\right] I_{\varepsilon}+\left[\int_{0}^{1}\left(b_{x}(\bar{x}+\lambda \Delta x, \bar{u})-b_{x}(\bar{x}, \bar{u})\right) d \lambda\right]^{T} \Delta x\right| \\
& \leq C\left[\psi_{\varepsilon}(t) I_{\varepsilon}(t)+|\Delta x(t)|^{2}\right] .
\end{aligned}
$$

Similarly,

$$
\left|\Delta_{x u}^{1} \sigma^{i}(t)\right| \leq C\left[\psi_{\varepsilon}(t) I_{\varepsilon}(t)+|\Delta x(t)|^{2}\right] .
$$

Hence, by $(4.22)$ with $\left(\theta_{1}, \theta_{2}, \theta_{3}, \theta_{4}, \lambda\right)=(1,1,1,1,1)$ and $(0,1,3,1,0)$, respectively, we have

$$
\begin{aligned}
& \left|\mathbb{E} \int_{0}^{T} \Delta x(t)^{T}\left[P^{\ell}(t) \Delta_{x u}^{1} b(t)+\sum_{i=1}^{d} Q_{i}^{\ell}(t) \Delta_{x u}^{1} \sigma^{i}(t)\right] d t\right| \\
& \leq C\left[\mathbb{E} \int_{0}^{T} \psi_{\varepsilon}(t) \eta(t)|\Delta x(t)| I_{\varepsilon}(t) d t+\mathbb{E} \int_{0}^{T} \eta(t)|\Delta x(t)|^{3} d t\right] \\
& =C\left[o(1) \varepsilon+\varepsilon^{\frac{3}{2}}\right]=o(\varepsilon),
\end{aligned}
$$

proving (4.30). 
Proof of (4.31): We let

$$
\begin{aligned}
R^{i}(t) & \triangleq \Delta_{x u}^{0} \sigma^{i}(t)-\Delta_{u} \sigma^{i}\left(t, u_{\varepsilon}(t)\right) I_{\varepsilon}(t)-\sigma_{x}^{i}(t) \Delta x(t) \\
& =\sigma^{i}\left(x_{\varepsilon}, u_{\varepsilon}\right)-\sigma^{i}(\bar{x}, \bar{u})-\sigma^{i}\left(\bar{x}, u_{\varepsilon}\right)+\sigma^{i}(\bar{x}, \bar{u})-\sigma_{x}^{i}(\bar{x}, \bar{u}) \Delta x \\
& =\int_{0}^{1}\left[\sigma_{x}^{i}\left(\bar{x}+\lambda \Delta x, u_{\varepsilon}\right)-\sigma_{x}^{i}(\bar{x}, \bar{u})\right]^{T} \Delta x d \lambda
\end{aligned}
$$

By assumption (S2), $\left|R^{i}(t)\right| \leq 2 L|\Delta x(t)|$. Therefore we have (with arguments suppressed)

$$
\begin{aligned}
& \mathbb{E} \int_{0}^{T}\left|\left(\Delta_{x u}^{0} \sigma\right)^{T} P^{\ell} \Delta_{x u}^{0} \sigma^{i}-\left(\sigma_{x}^{i} \Delta x\right)^{T} P^{\ell} \sigma_{x}^{i} \Delta x-\left(\Delta_{u} \sigma^{i}\right)^{T} P^{\ell}\left(\Delta_{u} \sigma^{i}\right) I_{\varepsilon}\right| d t \\
& \leq \mathbb{E} \int_{0}^{T} \mid\left(\Delta_{u} \sigma^{i} I_{\varepsilon}+\sigma_{x}^{i} \Delta x+R^{i}\right)^{T} P^{\ell}\left(\Delta_{u} \sigma^{i} I_{\varepsilon}+\sigma_{x}^{i} \Delta x+R^{i}\right) \\
& -\left(\sigma_{x}^{i} \Delta x\right)^{T} P^{\ell} \sigma_{x}^{i} \Delta x-\left(\Delta_{u} \sigma^{i}\right)^{T} P^{\ell}\left(\Delta_{u} \sigma^{i}\right) I_{\varepsilon} \mid d t \\
& \leq \mathbb{E} \int_{0}^{T}\left|P^{\ell}\right|\left[2\left|\Delta_{u} \sigma^{i}\right|\left(\left|\sigma_{x}^{i} \Delta x\right|+\left|R^{i}\right|\right) I_{\varepsilon}+\left(2\left|\sigma_{x}^{i} \Delta x\right|+\left|R^{i}\right|\right)\left|R^{i}\right|\right] d t \\
& \leq \mathbb{E} \int_{0}^{T} \eta\left[6 L \psi_{\varepsilon}|\Delta x| I_{\varepsilon}+4 L|\Delta x|\left|R^{i}\right|\right] d t .
\end{aligned}
$$

To finish the proof, another bound for $\left|R^{i}(t)\right|$ is needed. Rewrite $R^{i}(t)$ as follows.

$$
R^{i}(t)=\int_{0}^{1}\left[\sigma_{x}^{i}\left(\bar{x}+\lambda \Delta x, u_{\varepsilon}\right)-\sigma_{x}^{i}(\bar{x}+\lambda \Delta x, \bar{u})+\sigma_{x}^{i}(\bar{x}+\lambda \Delta x, \bar{u})-\sigma_{x}^{i}(\bar{x}, \bar{u})\right]^{T} \Delta x d \lambda .
$$

For a fixed $\tau \in(0, \min \{\beta-3,1\})$, by assumption (S2), we have

$$
\left|\sigma_{x}^{i}(\bar{x}+\lambda \Delta x, \bar{u})-\sigma_{x}^{i}(\bar{x}, \bar{u})\right| \leq \min \left\{2 L|\Delta x(t)|, L|\Delta x(t)|^{2}\right\} \leq 2 L|\Delta x(t)|^{1+\tau} .
$$

Thus, by (4.44) and (4.45), we obtain another bound for $R^{i}(t)$ :

$$
\left|R^{i}(t)\right| \leq 2 L\left(|\Delta x(t)| I_{\varepsilon}(t)+|\Delta x(t)|^{1+\tau}\right) .
$$

Substituting (4.46) into (4.43), and using (4.22) with $\left(\theta_{1}, \theta_{2}, \theta_{3}, \theta_{4}, \lambda\right)=(1,1,1,1,1)$, $(0,1,2,1,1)$ and $(0,1,2+\tau, 1,0)$, respectively, we obtain

$$
\begin{aligned}
& \mathbb{E} \int_{0}^{T}\left|\left(\Delta_{x u}^{0} \sigma\right)^{T} P^{\ell} \Delta_{x u}^{0} \sigma^{i}-\left(\sigma_{x}^{i} \Delta x\right)^{T} P^{\ell} \sigma_{x}^{i} \Delta x-\left(\Delta_{u} \sigma^{i}\right)^{T} P^{\ell}\left(\Delta_{u} \sigma^{i}\right) I_{\varepsilon}\right| d t \\
& \leq C \mathbb{E}\left(\int_{0}^{T}\left[\psi_{\varepsilon}(t) \eta(t)|\Delta x(t)| I_{\varepsilon}(t)+\eta(t)|\Delta x(t)|^{2} I_{\varepsilon}(t)+\eta(t)|\Delta x(t)|^{2+\tau}\right] d t\right) \\
& \leq C\left[o(1) \varepsilon+\varepsilon^{\frac{3}{2}}+\varepsilon^{\frac{2+\tau}{2}}\right]=o(\varepsilon) .
\end{aligned}
$$

This proves (4.31). 


\section{Pontryagin Type Extreme Principles}

In this section we apply the directional derivative formula established in the previous section to optimal controls and equilibria of differential games.

We first look at the case $N=1$, i.e., the classical stochastic optimal control problem which can be stated as follows.

Problem (C). Find $\bar{u}(\cdot) \in \mathcal{U}^{\beta}[0, T]$ such that

$$
J(\bar{u}(\cdot))=\min _{u(\cdot) \in \mathcal{U}^{\beta}[0, T]} J(u(\cdot)) .
$$

Any $\bar{u}(\cdot) \in \mathcal{U}^{\beta}[0, T]$ satisfying the above is called an optimal control of Problem (C), the corresponding state process $\bar{x}(\cdot)$ is called an optimal state process, and $(\bar{x}(\cdot), \bar{u}(\cdot))$ is called an optimal pair. The following result is referred to as Pontryagin type maximum principle, which is a set of first order necessary conditions for optimal controls. For optimal controls satisfying (4.5), the maximum principle is proved in [12]. Assuming more restrictive growth conditions for $f$ and $h$, the maximum principle is proved in [17]. See [3], [5], [7], [10], [13], [14], and [18] for related works.

Theorem 5.1. Let (S1)-(S2) hold with $N=1$. Suppose $\bar{u}(\cdot) \in \mathcal{U}^{\beta}[0, T]$ is an optimal control with $\beta>3$. Then there exist $(p(\cdot), q(\cdot))$ and $(P(\cdot), Q(\cdot))$ satisfying (3.2) and (3.3) (with $N=1$ ) such that

$$
\begin{aligned}
& \mathcal{H}(t, v) \triangleq H(t, \bar{x}(t), v, p(t), q(t))-H(t, \bar{x}(t), \bar{u}(t), p(t), q(t)) \\
& +\frac{1}{2} \sum_{i=1}^{d}\left[\sigma^{i}(t, \bar{x}(t), v)-\sigma^{i}(t, \bar{x}(t), \bar{u}(t))\right]^{T} P(t)\left[\sigma^{i}(t, \bar{x}(t), v)-\sigma^{i}(t, \bar{x}(t), \bar{u}(t))\right] \geq 0, \\
& \forall v \in U, \text { a.e.t } \in[0, T], \mathbb{P} \text {-a.s., }
\end{aligned}
$$

that is,

$$
\min _{v \in U} \mathcal{H}(t, v)=\mathcal{H}(t, \bar{u}(t))=0, \quad \text { a.e. } t \in[0, T], \mathbb{P} \text {-a.s. }
$$

Proof. Let us suppress $(t, \bar{x}(t), p(t), q(t))$. For any $v(\cdot) \in \mathcal{U}^{\beta}[0, T]$, by Theorem 4.1 , we have

$$
0 \leq \mathbb{E} \int_{0}^{T}\left\{H(v(t))-H(\bar{u}(t))+\frac{1}{2} \sum_{i=1}^{d}\left[\sigma^{i}(v(t))-\sigma^{i}(\bar{u}(t))\right]^{T} P(t)\left[\sigma^{i}(v(t))-\sigma^{i}(\bar{u}(t))\right]\right\} d t .
$$

Now if (5.2) fails, then there would be a $v_{0} \in U$ such that for some $\delta>0$, the set

$$
K \triangleq\left\{(t, \omega) \in[0, T] \times \Omega \mid \mathcal{H}\left(t, v_{0}, \omega\right) \leq-\delta\right\} \subseteq[0, T] \times \Omega
$$


has the property that

$$
\int_{0}^{T} \mathbb{P}\left(K_{t}\right) d t>0
$$

where

Now we let

$$
K_{t}=\{\omega \in \Omega \mid(t, \omega) \in K\} \in \mathcal{F}_{t}, \quad t \in[0, T] .
$$

$$
v(t, \omega)=\left\{\begin{array}{lc}
v_{0}, & (t, \omega) \in K, \\
\bar{u}(t, \omega), & \text { otherwise. }
\end{array}\right.
$$

Clearly, $v(\cdot) \in \mathcal{U}^{\beta}[0, T]$, and with this $v(\cdot)$, we have a violation of (5.4).

Putting the corresponding state equation, the first and second adjoint equations together, we obtain the following optimality system for an optimal control:

$$
\left\{\begin{array}{l}
d \bar{x}(t)=b(t, \bar{x}(t), \bar{u}(t)) d t+\sigma(t, \bar{x}(t), \bar{u}(t)) d W(t), \\
d p(t)=-H_{x}(t, \bar{x}(t), \bar{u}(t), p(t), q(t)) d t+q(t) d W(t), \\
d P(t)=-\left\{H_{x x}(t)+P(t) b_{x}(t)+b_{x}(t)^{T} P(t)\right. \\
\left.\quad+\sum_{i=1}^{d}\left[\sigma_{x}^{i}(t)^{T} P(t) \sigma_{x}^{i}(t)+Q_{i}(t) \sigma_{x}^{i}(t)+\sigma_{x}^{i}(t)^{T} Q_{i}(t)\right]\right\} d t+\sum_{i=1}^{d} Q_{i}(t) d W_{i}(t), \\
\begin{array}{rl}
x(0)=x_{0}, \quad p(T)=h_{x}(\bar{x}(T)), & P(T)=h_{x x}(\bar{x}(T)), \\
\min _{v \in U} \mathcal{H}(t, v)=0, \quad t \in[0, T], & \text { a.s. }
\end{array}
\end{array}\right.
$$

Note that the above is a coupled forward-backward stochastic differential equation (FBSDE, for short), with the coupling presented in the last minimum condition.

Next, we consider the case $N \geq 2$. This is an $N$-person stochastic differential game. There are a lot of situations in the area that one can discuss; see [1] for a general exposition. Here, we only consider the so-called non-cooperative differential games, namely, the $\ell$-th player in the game would like to minimize his/her own cost functional $J^{\ell}(u(\cdot)$, regardless of other players' cost functionals. To make it precise, let

$$
u(\cdot)=\left(u_{1}(\cdot), \cdots, u_{N}(\cdot)\right),
$$

with

$$
u_{\ell}(\cdot) \in \mathcal{U}_{\ell}^{\beta_{\ell}}[0, T] \triangleq L_{\mathcal{F}}^{\beta_{\ell}}\left(\Omega ; L^{2}\left(0, T ; U_{\ell}\right), \quad U_{\ell} \subseteq \mathbb{R}^{m_{\ell}}, \quad 1 \leq \ell \leq N .\right.
$$

For notational convenience, we introduce

$$
\left(u_{\ell}^{c}, v\right) \triangleq\left(u_{1}, \cdots, u_{\ell-1}, v, u_{\ell+1}, \cdots, u_{N}\right), \quad 1 \leq \ell \leq N .
$$

Then Player $\ell$ selects $u_{\ell}(\cdot) \in \mathcal{U}_{\ell}^{\beta_{\ell}}[0, T]$ to minimize the functional

$$
v(\cdot) \mapsto J^{\ell}\left(u_{\ell}^{c}(\cdot), v(\cdot)\right) \equiv J^{\ell}\left(u_{1}(\cdot), \cdots, u_{\ell-1}(\cdot), v(\cdot), u_{\ell+1}(\cdot), \cdots, u_{N}(\cdot)\right) .
$$


Obviously, $J^{\ell}(u(\cdot))$ not only depends on $u_{\ell}(\cdot)$, but also depends on $u_{k}(\cdot), k \neq \ell$. Hence, the optimal control/strategy of Player $\ell$ depends on the controls/stragies of other players. Therefore, we need to introduce the following notion.

Definition 5.1. An $N$-tuple $\bar{u}(\cdot) \equiv\left(\bar{u}_{1}(\cdot), \cdots, \bar{u}_{N}(\cdot)\right) \in \prod_{\ell=1}^{N} \mathcal{U}_{\ell}^{\beta_{\ell}}[0, T]$ is called an open-loop Nash equilibrium of the game if the following holds:

$$
J^{\ell}(\bar{u}(\cdot)) \leq J^{\ell}\left(\bar{u}_{\ell}^{c}(\cdot), v(\cdot)\right), \quad \forall v(\cdot) \in \mathcal{U}_{\ell}^{\beta_{\ell}}[0, T], 1 \leq \ell \leq N .
$$

Similar to Theorem 5.1, we have the following Pontryagin type maximum principle for Nash equilibria of the $N$-person stochastic differential game.

Theorem 5.2. Let (S1)-(S2) hold. Suppose $\bar{u}(\cdot) \equiv\left(\bar{u}_{1}(\cdot), \cdots, \bar{u}_{N}(\cdot)\right) \in \prod_{\ell=1}^{N} \mathcal{U}_{\ell}^{\beta_{\ell}}[0, T]$ is a Nash equilibrium of the game with each $\beta_{\ell}>3$. Then there exist $\left\{\left(p^{\ell}(\cdot), q^{\ell}(\cdot)\right), 1 \leq\right.$ $\ell \leq N\}$ and $\left\{\left(P^{\ell}(\cdot), Q^{\ell}(\cdot)\right), 1 \leq \ell \leq N\right\}$ satisfying (3.2) and (3.3) such that

$$
\begin{gathered}
\mathcal{H}^{\ell}(t, v) \triangleq H^{\ell}\left(t, \bar{x}(t), \bar{u}_{\ell}^{c}(t), v, p^{\ell}(t), q^{\ell}(t)\right)-H(t, \bar{x}(t), \bar{u}(t), p(t), q(t)) \\
+\frac{1}{2} \sum_{i=1}^{d}\left[\sigma^{i}\left(t, \bar{x}(t), \bar{u}_{\ell}^{c}(t), v\right)-\sigma^{i}(t, \bar{x}(t), \bar{u}(t))\right]^{T} P^{\ell}(t) \\
{\left[\sigma^{i}\left(t, \bar{x}(t), \bar{u}_{\ell}^{c}(t), v\right)-\sigma^{i}(t, \bar{x}(t), \bar{u}(t))\right] \geq 0,} \\
\forall v \in U_{\ell}, \text { a.e. } t \in[0, T], \mathbb{P} \text {-a.s., } 1 \leq \ell \leq N,
\end{gathered}
$$

that is,

$$
\min _{v \in U_{\ell}} \mathcal{H}^{\ell}\left(t, \bar{u}_{\ell}^{c}(t), v\right)=\mathcal{H}^{\ell}(t, \bar{u}(t))=0, \quad \text { a.e. } t \in[0, T], \mathbb{P} \text {-a.s. }
$$

The proof is very similar to that of Theorem 5.1, because for any $1 \leq \ell \leq N$, when $\bar{u}_{k}(\cdot)(k \neq \ell)$ are given, $\bar{u}_{\ell}(\cdot)$ is an optimal control problem with the state equation (1.1) in which $u(\cdot)=\left(\bar{u}_{\ell}^{c}(\cdot), v(\cdot)\right)$ and cost functional $J^{\ell}\left(\bar{u}_{\ell}^{c}(\cdot), v(\cdot)\right)$. Hence, Theorem 5.1 applies.

We point out that condition (5.7) gives couplings among $\left(\bar{u}_{\ell}(\cdot), p^{\ell}(\cdot), q^{\ell}(\cdot), P^{\ell}(\cdot)\right)$ for different $1 \leq \ell \leq N$. Similar to the case $N=1$, we may also write down the optimality system for $(\bar{x}(\cdot), \bar{u}(\cdot)),\left\{\left(p^{\ell}(\cdot), q^{\ell}(\cdot)\right), 1 \leq \ell \leq N\right\}$, and $\left\{\left(P^{\ell}(\cdot), Q^{\ell}(\cdot)\right), 1 \leq \ell \leq N\right\}$. Due to the minimum condition (5.8), it is not hard for us to see that the optimality system is not decoupled (with respect to $1 \leq \ell \leq N$ ). In fact, if for given for $t \in[0, T]$, $\left(\bar{u}_{1}(t), \cdots, \bar{u}_{N}(t)\right)$ is a solution of $(5.8)$, then we expect that

$$
\bar{u}_{\ell}(t)=\Phi_{\ell}\left(t, \bar{x}(t), p^{1}(t) \cdots, p^{N}(t), q^{1}(t), \cdots, q^{N}(t), P^{1}(t), \cdots, P^{N}(t)\right), \quad \ell=1, \cdots, N .
$$

Therefore, plugging the above into (1.1), (3.2), and (3.3), we obtain a couple system of FBSDEs. Solving that system will lead to a candidate for the Nash equilibria of the game. We prefer to omit the details here. 
Now let us look at an interesting special case: the two-person zero-sum differential games. Thus, $N=2$ and (1.3) holds. In this case, as we know that if $\left(\bar{u}_{1}(\cdot), \bar{u}_{2}(\cdot)\right) \in$ $\mathcal{U}^{\beta_{1}}[0, T] \times \mathcal{U}^{\beta_{2}}[0, T]$ is a Nash equilibrium, then

$$
\begin{cases}J^{1}\left(\bar{u}_{1}(\cdot), \bar{u}_{2}(\cdot)\right) \leq J^{1}\left(u_{1}(\cdot), \bar{u}_{2}(\cdot)\right), & \forall u_{1}(\cdot) \in \mathcal{U}_{1}^{\beta_{1}}[0, T], \\ J^{2}\left(\bar{u}_{1}(\cdot), \bar{u}_{2}(\cdot)\right) \leq J^{2}\left(\bar{u}_{1}(\cdot), u_{2}(\cdot)\right), & \forall u_{2}(\cdot) \in \mathcal{U}_{2}^{\beta_{2}}[0, T] .\end{cases}
$$

Due to $(1.3)$, we have (denoting $\left.J^{1}(u(\cdot))=\bar{J}(u(\cdot))\right)$

$$
\begin{aligned}
& \bar{J}\left(\bar{u}_{1}(\cdot), u_{2}(\cdot)\right) \leq \bar{J}\left(\bar{u}_{1}(\cdot), \bar{u}_{2}(\cdot)\right) \leq \bar{J}\left(u_{1}(\cdot), \bar{u}_{2}(\cdot)\right), \\
& \forall\left(u_{1}(\cdot), u_{2}(\cdot)\right) \in \mathcal{U}_{1}^{\beta_{1}}[0, T] \times \mathcal{U}_{2}^{\beta_{2}}[0, T] .
\end{aligned}
$$

Therefore, $\left(\bar{u}_{1}(\cdot), \bar{u}_{2}(\cdot)\right)$ is referred to as a saddle point of the game. For such a case, we have the following result.

Theorem 5.3. Suppose $\left(\bar{u}_{1}, \bar{u}_{2}(\cdot)\right) \in \mathcal{U}_{1}^{\beta_{1}}[0, T] \times \mathcal{U}_{2}^{\beta_{2}}[0, T]$ is a saddle point with $\beta_{1}, \beta_{2}>$ 3. Then there exist $(p(\cdot), q(\cdot))$ and $(P(\cdot), Q(\cdot))$ satisfying (3.2) and (3.3) (with $N=1$ ) such that with

$$
\begin{gathered}
\mathcal{H}\left(t, u_{1}, u_{2}\right) \triangleq f\left(t, \bar{x}(t), u_{1}, u_{2}\right)+p(t) \cdot b\left(t, \bar{x}(t), u_{1}, u_{2}\right)+\sum_{i=1}^{d} q_{i}(t) \cdot \sigma^{i}\left(t, \bar{x}(t), u_{1}, u_{2}\right) \\
+\frac{1}{2} \sum_{i=1}^{d}\left[\sigma^{i}\left(t, \bar{x}(t), u_{1}, u_{2}\right)-\sigma^{i}(t, \bar{x}(t), \bar{u}(t))\right]^{T} P(t)\left[\sigma^{i}\left(t, \bar{x}(t), u_{1}, u_{2}\right)-\sigma^{i}(t, \bar{x}(t), \bar{u}(t))\right], \\
\forall\left(u_{1}, u_{2}\right) \in U_{1} \times U_{2}, t \in[0, T],
\end{gathered}
$$

the following holds:

$$
\begin{array}{r}
\mathcal{H}\left(t, \bar{u}_{1}(t), u_{2}\right) \leq \mathcal{H}\left(t, \bar{u}_{1}(t), \bar{u}_{2}(t)\right) \leq \mathcal{H}\left(t, u_{1}, \bar{u}_{2}(t)\right), \\
\left(u_{1}, u_{2}\right) \in U_{1} \times U_{2}, \text { a.e.t } \in[0, T], \text { a.s. }
\end{array}
$$

The proof is pretty straightforward. We may refer (5.13) as a minimax condition.

\section{Sufficient Conditions for Nash Equilibria}

In this section, we use the first order representation in Lemma 3.1 to prove a sufficient condition for

$$
\bar{u}(\cdot) \equiv\left(\bar{u}_{1}(\cdot), \cdots, \bar{u}_{N}(\cdot)\right) \in \prod_{\ell=1}^{N} \mathcal{U}_{\ell}^{\beta_{\ell}}[0, T]
$$

to be a Nash equilibrium of the nonzero-sum game in Theorem 5.2. The proof follow directly from a generalization of the sufficient condition for optimal control proved in $[17]$. 
We need the concept of partial subgradient. For a function $\varphi$ defined on a convex subset $D$ of $\mathbb{R}^{n} \times \mathbb{R}^{m}$, the partial subgradient $\partial_{u} \varphi$ of $\varphi$ at $(x, u) \in D$ is defined as

$$
\begin{aligned}
& \partial_{u} \varphi(x, u) \\
& =\left\{\eta \in \mathbb{R}^{m} \mid \eta \cdot y \leq \lim _{r \rightarrow 0+} \sup \frac{1}{r}[\varphi(x, u+r y)-\varphi(x, u)] \text { for each } y \in \mathbb{R}^{m}\right\} .
\end{aligned}
$$

See Lemma 2.3, Chapter 3 in [17] for properties of $\partial_{u} \varphi$. We make the following assumption.

S1*. The control domain $U$ is a convex body in $\mathbb{R}^{m}$.

S2*. (i) The maps $b, \sigma, f$ and $h$ satisfy (i) and (ii) in assumption (S2).

(ii) For almost all $(t, u, \omega) \in[0, T] \times \mathbb{R}^{n} \times \Omega$, the map

$$
x \mapsto(b(t, x, u, \omega), \sigma(t, x, u, \omega), f(t, x, u, \omega), h(x, \omega))
$$

is continuously differentiable with appropriate growths. More precisely, there exist a constant $L>0$, a process $\rho(\cdot) \in L_{\mathcal{F}}^{\beta}\left(\Omega ; L^{2}(0, T ; \mathbb{R})\right.$ ) (with $\beta \geq 1$ ) and a modulus of continuity $\widetilde{\omega}:[0, \infty) \rightarrow[0,1]$ such that $\varphi=b, \sigma$ and $\psi=f, h$ satisfy

$$
\begin{aligned}
& \left|\varphi_{x}(t, x, u)-\varphi_{x}(t, \widehat{x}, \widehat{u})\right| \leq L \widetilde{\omega}(|x-\widehat{x}|+|u-\widehat{u}|), \\
& \left|\psi_{x}(t, x, u)-\psi_{x}(t, \widehat{x}, \widehat{u})\right| \\
& \leq L(\rho(t)+|x|+|\widehat{x}|+|u|+|\widehat{u}|) \widetilde{\omega}(|x-\widehat{x}|+|u-\widehat{u}|),
\end{aligned}
$$

for all $t \in[0, T], x, \widehat{x} \in \mathbb{R}^{n}$ and $u, \widehat{u} \in U$.

Now we prove

Theorem 6.1. (Sufficient conditions of optimality). Let $S 1^{*}$ and $S 2^{*}$ hold. Suppose that $\bar{u}(\cdot) \in L_{\mathcal{F}}^{2}(0, T ; U)$ with adjoint processes $(p(t), q(t))$ defined by $(3.2)$ and $\bar{u}(\cdot)$ satisfies, for all $t \in[0, T]$,

(i) $h(x)$ is convex in $x$ and $H(t, x, u, p(t), q(t))$ is convex in $x$ and $u$.

(ii) $0 \in \partial_{u} H(t, \bar{x}(t), \bar{u}(t), p(t), q(t))$.

Then $\bar{u}(t)$ is an optimal control.

Proof. Let $(\bar{x}(t), \bar{u}(t))$ be an admissible pair with $\bar{u}(t)$ in Theorem 6.1 and $(p(\cdot), q(\cdot))$ being the associated first order adjoint processes. Let $(x(t), u(t))$ be any admissible pair.

Note that assumption $\left(\mathrm{S} 2^{*}\right)$ is sufficient for deriving the first order representation in Lemma 3.1, which can be written as

$$
\begin{aligned}
& J(u)-J(\bar{u}) \\
& =\mathbb{E}\left\{\Delta_{x x} h(\bar{x}(T))+\int_{0}^{T}\left[H(x, u)-H(\bar{x}, \bar{u})-H_{x}(\bar{x}, \bar{u}) \cdot \Delta x\right] d t\right\},
\end{aligned}
$$

where we have dropped $(t, p(t), q(t))$ from notations, and $\Delta x=x-\bar{x}$. For example,

$$
H(x, u)-H(\bar{x}, \bar{u})=H(t, x(t), u(t), p(t), q(t))-H(t, \bar{x}(t), \bar{u}(t), p(t), q(t)) .
$$


The convexity of $h$ implies

$$
\Delta_{x x} h(\bar{x}(T))=h(x(T))-h(\bar{x}(T))-h_{x}(\bar{x}(T)) \cdot \Delta \bar{x}(T) \geq 0 .
$$

Thus, to prove that $\bar{u}$ is an optimal control, it suffices to show that for all $t \in[0, T]$,

$$
H(x, u)-H(\bar{x}, \bar{u})-H_{x}(\bar{x}, \bar{u}) \cdot \Delta x \geq 0 .
$$

The convexity of $H(t, \cdot, \cdot, p(t), q(t))$ implies that the functions

$$
\begin{aligned}
& g(r) \triangleq \frac{1}{r}[H(\bar{x}+r \Delta x, \bar{u}+r \Delta u)-H(\bar{x}, \bar{u})], \\
& g_{1}(r) \triangleq \frac{\Delta}{r}[H(\bar{x}, \bar{u}+r \Delta u)-H(\bar{x}, \bar{u})],
\end{aligned}
$$

are increasing in $r \in[0,1]$ (for each $t$ ). By condition (ii) and assumption $(\mathrm{S} 2 *$ ), for a.e. and a.s. $(t, \omega) \in[0, T] \times \Omega$, the following hold.

$$
\begin{aligned}
& \lim _{r \rightarrow 0+} g_{1}(r) \text { exists and } \geq 0, \\
& \lim _{r \rightarrow 0+}\left(g(r)-g_{1}(r)\right)=\lim _{r \rightarrow 0+} \frac{1}{r}[H(\bar{x}+r \Delta x, \bar{u}+r \Delta u)-H(\bar{x}, \bar{u}+r \Delta u)] \\
& =\lim _{r \rightarrow 0+} \int_{0}^{1} H_{x}(\bar{x}+r s \Delta x, \bar{u}+r \Delta u) \Delta x d s=H_{x}(\bar{x}, \bar{u}) \cdot \Delta x, \\
& \lim _{r \rightarrow 0+} g(r)=H_{x}(\bar{x}, \bar{u}) \cdot \Delta x+\lim _{r \rightarrow 0+} g_{1}(r) \geq H_{x}(\bar{x}, \bar{u}) \cdot \Delta x .
\end{aligned}
$$

Recall that $g(r)$ is decreasing as $r \rightarrow 0+$. Therefore,

$$
H(x, u)-H(\bar{x}, \bar{u})=g(1) \geq g(r) \geq \lim _{r \rightarrow 0+} g(r) \geq H_{x}(\bar{x}, \bar{u}) \cdot \Delta x .
$$

This finishes the proof.

We remark that condition (ii) in Theorem 6.1 can be verified easily in certain cases. Clearly if $\bar{u}(t)$ is a minimum point of $H(t, \bar{x}(t), u, p(t), q(t))$ for every $t$, then condition (ii) holds; see Lemma 2.3 (iii) in Section 3.2 of [17].

On the other hand, if $\bar{u}(t)$ is a minimum point of $\mathcal{H}(t, u)$ for every $t \in[0, T]$ (this is the necessary condition in Theorem 5.1), then $0 \in \partial_{u} \mathcal{H}(t, \bar{u}(t)$ ) (see Lemma 2.3 (iii) in [17], Section 3.2). If, in addition, $\sigma(t, \bar{x}(t), u)$ is $C^{\alpha}$ in $u$ with $\alpha>\frac{1}{2}$, then we will have that $0 \in \partial_{u} H(t, \bar{x}(t), u, p(t), q(t))$. So condition (ii) also holds.

Now a sufficient condition for the nonzero-sum game in Theorem 5.2 can be easily derived.

Theorem 6.2. (Sufficient conditions of Nash equilibria). Let $S 1^{*}$ and $S 2^{*}$ hold. Suppose $\bar{u}(\cdot) \equiv\left(\bar{u}_{1}(\cdot), \cdots, \bar{u}_{N}(\cdot)\right) \in \prod_{\ell=1}^{N} \mathcal{U}_{\ell}^{\beta_{\ell}}[0, T]$ and $\left(p^{\ell}, q^{\ell}\right)$ are the adjoint processes for Player $\ell, 1 \leq \ell \leq N$. Assume that

(i) $h^{\ell}(x)$ is convex in $x$ for $1 \leq \ell \leq N$.

(ii) $H^{\ell}\left(t, x, v, u_{\ell}^{c}, p^{\ell}(t), q^{\ell}(t)\right)$ is convex in $(x, v)$ for $t \in[0, T]$.

(iii) $0 \in \partial_{u_{\ell}} H^{\ell}\left(t, \bar{x}(t), \bar{u}_{\ell}, p^{\ell}(t), q^{\ell}(t)\right)$. 
Then $\bar{u}(\cdot) \equiv\left(\bar{u}_{1}(\cdot), \cdots, \bar{u}_{N}(\cdot)\right)$ is a Nash equilibrium point of the nonzero-sum game.

Proof. We apply Theorem 6.1 to $J^{\ell}\left(\bar{u}_{\ell}^{c}(\cdot), v(\cdot)\right)$ to conclude that $v(\cdot)=\bar{u}_{\ell}(\cdot)$ is an optimal control of $J^{\ell}\left(\bar{u}_{\ell}^{c}(\cdot), v(\cdot)\right)$.

\section{REFERENCES}

[1] T. Basar and G.J. Olsder, Dynamic Noncooperative Game Theory, 2nd Edition, Academic Press, London/New York, 1995.

[2] A. Bensoussan, Lectures on Stochastic Control, in Lecture Notes in Mathematics, 972, SpringerVerlag, Berlin, 1981.

[3] F. Biagini, Y. Hu, B. O. Ksendal, A. Sulem, A stochastic maximum principle for processes driven by fractional Brownian motion, Stochastic Process. Appl. 100 (2002), 233-253.

[4] J. M. Bismut, Conjugate convex functions in optimal stochastic control, J. Math. Anal.Appl. 44 (1973) 384-404.

[5] A. Candenillas and I. Karatzas, The Stochastic maximum principle for linear optimal control with random coefficients, SIAM J. Control Optim. 33 (1995), No. 2, 590-624.

[6] N. El Karoui, S. Peng, and M. C. Quenez, Backward stochastic differential equations in finance, Math. Finance, 7 (1997), 1-71.

[7] W.H. Fleming and R.W. Rischel, Deterministic and Stochastic Optimal Control, Springer-Verlag, New York, 1975.

[8] F. Gozzi, A. Swiech and X.Y. Zhou, A corrected proof of the stochastic verification theorem with the framework of viscosity solutions, SIAM J. Control Optim., Vol. 43 (2005), No. 6, 2009-2019.

[9] X. Li and J. Yong, Optimal Control Theory for Infinite Dimensional Systems, Birkhäuser, Boston, 1995.

[10] N.I. Mahmudov, The maximum principle for stochastic evolution systems in Hilbert spaces, Int. J. Pure Appl. Math. 2 (2002), No. 3, 287-298.

[11] L. Mou and J.M. Yong, Two-person zero-sum linear quadratic stochastic differential games by a Hilbert space method, Journal of Industrial and Management Optimization (2006), Vol. 2, No. 1, 95-117.

[12] S. Peng, A general stochastic maximum principle for optimal control problems, SIAM J. Control \& Optim., 28 (1990), 966-979.

[13] A.S. Poznyak, B. Pasik-Duncan, T.E. Duncan, V.G. Boltyansky, Robust stochastic maximum principle for multi-model worst case optimization. Internat. J. Control 75 (2002), No. 13, 10321048.

[14] S. Tang, The maximum principle for partially observed optimal control of stochastic differential equations, SIAM J. Control Optim. 36 (1998), No. 5, 1596-1617.

[15] J. Yong, Pontryagin maximum principle for semilinear second order elliptic partial differential equations and variational inequalities with state constraints, Diff. Int. Eqs., 5 (1992), 1307-1334.

[16] J. Yong, A leader-follower stochastic linear quadratic differential game, SIAM J. Control and Optimization, Vol. 41, No. 4 (2002) 1015-1041.

[17] J. Yong and X.Y. Zhou, Stochastic Controls, Hamiltonian Systems and HJB Equations, Springer 1999.

[18] X.Y. Zhou, A unified treatment of maximum principle and dynamic programming in stochastic controls, Stochastics Stochastics Rep. 36 (1991), No. 3-4, 137-161.

Libin Mou

Department of Mathematics 
Bradley University, Peoria, IL 61625

E-mail: mou@hilltop.bradley.edu

Jiongmin Yong

Department of Mathematics

University of Central Florida, Orlando, FL 32816

E-mail: jyong@mail.ucf.edu 